\title{
Bifurcations from the orbit of solutions of the Neumann problem
}

\author{
Anna Gołębiewska1 $^{1}$ - Joanna Kluczenko² ${ }^{2}$. \\ Piotr Stefaniak ${ }^{3}$
}

Received: 14 April 2017 / Accepted: 8 December 2017 / Published online: 26 December 2017

(C) The Author(s) 2017. This article is an open access publication

\begin{abstract}
The purpose of this paper is to study weak solutions of a nonlinear Neumann problem considered on a ball. Assuming that the potential is invariant, we consider an orbit of critical points, i.e. we do not assume that critical points are isolated. We apply techniques of equivariant analysis to examine bifurcations from the orbits of trivial solutions. We formulate sufficient conditions for local and global bifurcations, in terms of the right-hand side of the system and eigenvalues of the Laplace operator. Moreover, we characterise orbits at which global symmetry breaking phenomena occur.
\end{abstract}

Mathematics Subject Classification Primary: 35B32 - Secondary: 35J20

\section{Introduction}

In this paper, we study bifurcations of weak solutions of elliptic systems of the form:

Communicated by P. Rabinowitz.

Anna Gołębiewska

Anna.Golebiewska@mat.umk.pl

Joanna Kluczenko

jgawrycka@matman.uwm.edu.pl

Piotr Stefaniak

pstefaniak@zut.edu.pl

1 Faculty of Mathematics and Computer Science, Nicolaus Copernicus University, ul. Chopina 12/18, 87-100 Toruń, Poland

2 Faculty of Mathematics and Computer Science, University of Warmia and Mazury, ul. Sloneczna 54, 10-710 Olsztyn, Poland

3 School of Mathematics, West Pomeranian University of Technology, al. Piastów 48/49, 70-310 Szczecin, Poland 


$$
\begin{cases}-\Delta u=\lambda \nabla F(u) & \text { in } B^{N} \\ \frac{\partial u}{\partial \nu}=0 & \text { on } S^{N-1}\end{cases}
$$

where $B^{N}$ is the open unit ball in $\mathbb{R}^{N}, S^{N-1}=\partial B^{N}$ and the function $F: \mathbb{R}^{m} \rightarrow \mathbb{R}$ satisfies additional assumptions, see Sect. 2.

In particular, we are interested in the equivariant case. Namely, we assume that on the space $\mathbb{R}^{m}$ there is defined an action of a compact Lie group $\Gamma$ and $\nabla F$ is a $\Gamma$-equivariant mapping. Moreover, it is known that $B^{N}$ is $S O(N)$-invariant, where $S O(N)$ stands for the special orthogonal group in dimension $N$.

Consider the set $\nabla F^{-1}(0)$. For $u_{0} \in \nabla F^{-1}(0)$ the constant function $\tilde{u}_{0} \equiv u_{0}$ is a solution of (1.1) for all $\lambda \in \mathbb{R}$. Therefore, we obtain the family of trivial solutions $\left\{\tilde{u}_{0}\right\} \times \mathbb{R}$. Investigating the change of the Conley index for different levels $\lambda \in \mathbb{R}$, one can obtain a sequence of nontrivial weak solutions bifurcating from the point $\left(\tilde{u}_{0}, \lambda_{0}\right)$, for some values $\lambda_{0} \in \mathbb{R}$. Investigating the change of the topological degree, one can prove the existence of the continuum, emanating from $\left(\tilde{u}_{0}, \lambda_{0}\right)$, of nontrivial weak solutions of the system (i.e. the global bifurcation of weak solutions).

For a system of elliptic differential equations with Dirichlet boundary conditions such methods have been used in many papers, among others by the first and the second author in $[7,10,15]$. A similar method has been also used in [9] for the system with Neumann boundary conditions for bifurcation from infinity instead from critical points. The phenomenon of symmetry breaking for elliptic systems with Neumann boundary conditions has been considered by the third author in [27].

The results described above are obtained with the assumption that $u_{0}$ is an isolated critical point of the potential $F$.

Assuming that $\nabla F$ is a $\Gamma$-equivariant mapping, we obtain that for $u_{0} \in \nabla F^{-1}(0)$ also $\gamma u_{0} \in \nabla F^{-1}(0)$ for all $\gamma \in \Gamma$. It is therefore clear, that the assumption that the critical point $u_{0}$ is an isolated one, does not have to be satisfied in this case.

The method, that can be used in this situation, is an investigation of the index of the isolated orbit. Under some additional assumptions, this method has been recently proposed by Pérez-Chavela et al. [18]. In that paper it has been proved that the computation of the Conley index of the orbit can be in some cases reduced to computation of the index of a point from the space normal to the orbit.

To study weak solutions of the system (1.1) we apply variational methods, i.e. we associate with the system a functional $\Phi$ defined on a suitable Hilbert space $\mathbb{H}$. Its critical points are in one-to-one correspondence with weak solutions of (1.1). The tools we use are the finite and infinite dimensional equivariant Conley index (see $[2,8]$ for the definition in the finite dimensional case and [13] for the infinite dimensional case) and the degree for equivariant gradient maps, defined in [22].

Consider the group $\mathcal{G}=\Gamma \times S O(N)$. Since $\mathbb{R}^{m}$ is a $\Gamma$-representation (by assumption) and $B^{N}$ is an $S O(N)$-invariant set, it follows that the space $\mathbb{H}$ is a $\mathcal{G}$-representation. Moreover, for $u_{0} \in(\nabla F)^{-1}(0),\left(g \tilde{u}_{0}, \lambda\right)$ is a critical point of $\Phi$ for all $g \in \mathcal{G}, \lambda \in \mathbb{R}$.

Therefore we can consider the set of trivial solutions $\mathcal{T}=\mathcal{G}\left(\tilde{u}_{0}\right) \times \mathbb{R}$. We are going to investigate bifurcations of nontrivial solutions from the family $\mathcal{T}$. Our aim is to formulate necessary and sufficient conditions, in terms of the right-hand side of the system and of the eigenvalues of the Laplace operator, for a bifurcation from the orbit $\mathcal{G}\left(\tilde{u}_{0}\right) \times\left\{\lambda_{0}\right\}$.

We also consider global symmetry breaking phenomena at the orbit $\mathcal{G}\left(\tilde{u}_{0}\right) \times\left\{\lambda_{0}\right\}$. More precisely, knowing that the trivial solutions are radial, we study when the bifurcating solutions are non-radial. The analogous problem has been studied by the third 
author in $[23,24]$ on the sphere and on the geodesic ball, with the use of a lemma due to Dancer (see [4]), characterising isotropy groups of bifurcating solutions. In our situation, if the group $\Gamma$ is not a discrete one, we cannot use this result. Therefore we generalise it.

After this introduction the paper is organised in the following way:

In Sect. 2 we introduce the problem and recall some definitions. With an elliptic system on a ball we associate a functional. Next we study the properties of the linear system. We end this section with the definitions of local and global bifurcations from an orbit and of the admissible pair.

In Sect. 3 we formulate and prove the main results of this article, namely Theorem 3.5 concerning the global bifurcation of solutions, and Theorem 3.10, concerning the symmetry breaking problem. First we consider the phenomenon of bifurcation from the critical orbit. We start with some auxiliary results. In Lemma 3.1 we describe the set of parameters at which the bifurcation of solutions can occur. In Theorem 3.3 we investigate the change of the Conley index at the levels obtained in Lemma 3.1. This result is applied to prove Theorem 3.5. Since the global bifurcation implies the local one, we obtain the result concerning local bifurcations, see Corollary 3.6. This phenomenon can be proved also directly from Theorem 3.3, what is described in Remark 3.7. The local bifurcation of solutions, under weaker assumptions, is considered also in Theorem 3.8. Next we study the symmetry breaking problem. In Theorem 3.10 we prove the bifurcation of orbits of non-radial solutions emanating from orbits of radial ones. To obtain this result, we generalise the result of Dancer in Lemma 3.11.

In Sect. 4 we illustrate our results with a few examples. Using the properties of the eigenspaces of the Laplace operator (with Neumann boundary conditions) on the ball, we verify the assumptions of our main results.

Section 5 is the "Appendix". In the main part of our paper we assume that the reader is familiar with some classical definitions and facts, concerning for example the equivariant Conley index, the degree for equivariant gradient maps or the properties of eigenspaces of the Laplace operator on a ball. However, it is not easy to find a detailed discussion of these properties. Therefore, for the completeness of the paper we collect in this section the information which we use to prove our main results. In this section we present also an equivariant version of the implicit function theorem in infinite dimensional spaces, due to Dancer.

\subsection{Notation}

Suppose that $G$ is a compact Lie group. We denote by $\overline{\operatorname{sub}}(G)$ the set of closed subgroups of $G$. For $u$ from a given $G$-space $X$ we denote by $G(u)$ the orbit through $u$ and $G_{u}$ stands for the isotropy group of $u$. By $X^{G}$ we denote the space of all fixed points of the action of the group $G$ on $X$.

Further, by $U(G)$ we denote the Euler ring of $G$ and we use the symbol $\chi_{G}(\cdot)$ to denote the $G$-equivariant Euler characteristic of a pointed finite $G$-CW-complex. Moreover, the symbols $C I_{G}(S, f)$ and $\mathcal{C I}_{G}(S, f)$ stand for the Conley indices of an isolated invariant set $S$ of the flow generated by $f$, considered respectively in finite and infinite dimensional cases. A more precise description can be found in the "Appendix".

Finally, for a Hilbert space $\mathbb{H}$ and $u_{0} \in \mathbb{H}$ we denote by $B_{\delta}\left(u_{0}, \mathbb{H}\right)\left(\right.$ respectively $D_{\delta}\left(u_{0}, \mathbb{H}\right)$ ) the open (respectively closed) ball in $\mathbb{H}$ centred at $u_{0}$ and with radius $\delta$. In particular, we use the symbol $B^{N}$ for the open ball if $\delta=1, u_{0}=0$ and $\mathbb{H}=\mathbb{R}^{N}$ and we write $S^{N-1}$ for $\partial B^{N}$. 


\section{Preliminaries}

Throughout this paper $\Gamma$ stands for a compact Lie group and $\mathbb{R}^{m}$ is an orthogonal representation of the group $\Gamma$. Consider $F: \mathbb{R}^{m} \rightarrow \mathbb{R}$ satisfying:

(B1) $F \in C^{2}\left(\mathbb{R}^{m}, \mathbb{R}\right)$ is such that for every $u \in \mathbb{R}^{m}$ we have $\left|\nabla^{2} F(u)\right| \leq a+b|u|^{q}$ where $a, b \in \mathbb{R}$ and $1<q<\frac{4}{N-2}$ for $N \geq 3$ and $1<q<\infty$ for $N=2$,

(B2) $F$ is $\Gamma$-invariant, i.e. $F(\gamma u)=F(u)$ for every $\gamma \in \Gamma, u \in \mathbb{R}^{m}$.

Our aim is to study bifurcations of weak solutions of the nonlinear Neumann problem, parameterised by $\lambda \in \mathbb{R}$,

$$
\left\{\begin{array}{ll}
-\triangle u=\lambda \nabla F(u) & \text { in } B^{N} \\
\frac{\partial u}{\partial v}=0 & \text { on } S^{N-1}
\end{array} .\right.
$$

Denote by $H^{1}\left(B^{N}\right)$ the usual Sobolev space on $B^{N}$ and consider the separable Hilbert space $\mathbb{H}=\bigoplus_{i=1}^{m} H^{1}\left(B^{N}\right)$ with the scalar product

$$
\langle v, w\rangle_{\mathbb{H}}=\sum_{i=1}^{m}\left\langle v_{i}, w_{i}\right\rangle_{H^{1}\left(B^{N}\right)}=\sum_{i=1}^{m} \int_{B^{N}}\left(\nabla v_{i}(x), \nabla w_{i}(x)\right)+v_{i}(x) \cdot w_{i}(x) d x .
$$

Denote by $\mathcal{G}$ the group $\Gamma \times S O(N)$, where $S O(N)$ is the special orthogonal group in dimension $N$. Note that the space $\mathbb{H}$ with the scalar product given by (2.2) is an orthogonal $\mathcal{G}$-representation with the $\mathcal{G}$-action given by

$$
(\gamma, \alpha)(u)(x)=\gamma u\left(\alpha^{-1} x\right) \text { for }(\gamma, \alpha) \in \mathcal{G}, u \in \mathbb{H}, x \in B^{N} .
$$

It is well known that weak solutions of the problem (2.1) are in one-to-one correspondence with critical points (with respect to $u$ ) of the functional $\Phi: \mathbb{H} \times \mathbb{R} \rightarrow \mathbb{R}$ defined by

$$
\Phi(u, \lambda)=\frac{1}{2} \int_{B^{N}}|\nabla u(x)|^{2} d x-\lambda \int_{B^{N}} F(u(x)) d x .
$$

Computing the gradient of $\Phi$ with respect to $u$ we obtain:

$$
\left\langle\nabla_{u} \Phi(u, \lambda), v\right\rangle_{\mathbb{H}}=\int_{B^{N}}(\nabla u(x), \nabla v(x))-(\lambda \nabla F(u(x)), v(x)) d x, u, v \in \mathbb{H} .
$$

Moreover,

$$
\begin{aligned}
\left\langle\nabla_{u}^{2} \Phi(u, \lambda) w, v\right\rangle_{\mathbb{H}}= & \int_{B^{N}}(\nabla w(x), \nabla v(x)) \\
& -\left(\lambda \nabla^{2} F(u(x)) w(x), v(x)\right) d x, u, w, v \in \mathbb{H} .
\end{aligned}
$$

Assumption (B2) implies that $\nabla_{u} \Phi: \mathbb{H} \times \mathbb{R} \rightarrow \mathbb{H}$ is $\mathcal{G}$-equivariant.

Moreover, from imbedding theorems and the assumption (B1) it follows that the operator $\nabla_{u} \Phi$ is a completely continuous perturbation of the identity, see [21].

\subsection{Linear equation}

In this subsection we consider the Eq. (2.1) in the linear case, i.e. the system:

$$
\begin{cases}-\triangle u=\lambda A u & \text { in } B^{N} \\ \frac{\partial u}{\partial v}=0 & \text { on } S^{N-1}\end{cases}
$$


where $A$ is a real, symmetric $(m \times m)$-matrix.

Using formula (2.4) we can associate with (2.6) the functional $\Phi_{A}: \mathbb{H} \times \mathbb{R} \rightarrow \mathbb{R}$ given by

$$
\Phi_{A}(u, \lambda)=\frac{1}{2} \int_{B^{N}}|\nabla u(x)|^{2} d x-\frac{\lambda}{2} \int_{B^{N}}(A u(x), u(x)) d x .
$$

Note that from (2.5) for every $v \in \mathbb{H}$ we have

$$
\left\langle\nabla_{u} \Phi_{A}(u, \lambda), v\right\rangle_{\mathbb{H}}=\langle u, v\rangle_{\mathbb{H}}-\left\langle L_{\lambda A} u, v\right\rangle_{\mathbb{H}},
$$

where

$$
\left\langle L_{\lambda A} u, v\right\rangle_{\mathbb{H}}=\int_{B^{N}}(u(x), v(x))+(\lambda A u(x), v(x)) d x .
$$

The existence and boundedness of the operator $L_{\lambda A}: \mathbb{H} \rightarrow \mathbb{H}$ follow from the Riesz theorem. By definition $L_{\lambda A}$ is self-adjoint.

Let us denote by $\sigma\left(-\Delta ; B^{N}\right)=\left\{0=\beta_{1}<\beta_{2}<\ldots<\beta_{k}<\ldots\right\}$ the set of distinct eigenvalues of the Laplace operator (with Neumann boundary conditions) on the ball. Write $\mathbb{V}_{-\Delta}\left(\beta_{k}\right)$ for the eigenspace of $-\Delta$ corresponding to $\beta_{k} \in \sigma\left(-\Delta ; B^{N}\right)$. In the "Appendix" we give a more precise description of these eigenspaces. By the spectral theorem it follows that $H^{1}\left(B^{N}\right)=\operatorname{cl}\left(\bigoplus_{k=1}^{\infty} \mathbb{V}_{-\Delta}\left(\beta_{k}\right)\right)$. Let us denote by $\mathbb{H}_{k}$ the space $\bigoplus_{i=1}^{m} \mathbb{V}_{-\Delta}\left(\beta_{k}\right)$. In particular, $u=\sum_{k=1}^{\infty} u_{k}$ for every $u \in \mathbb{H}$, where $u_{k} \in \mathbb{H}_{k}$.

Let $\alpha_{1}, \ldots, \alpha_{m}$ denote the eigenvalues of $A$ (not necessarily distinct) with corresponding eigenvectors $f_{1}, \ldots, f_{m}$, which form an orthonormal basis of $\mathbb{R}^{m}$.

Let $\pi_{j}: \mathbb{H} \rightarrow H^{1}\left(B^{N}\right)$ be the projection such that $\pi_{j}(u)(x)=\left(u(x), f_{j}\right), j=1, \ldots, m$. Clearly, if $u_{k} \in \mathbb{H}_{k}$, then $\pi_{j}\left(u_{k}\right) \in \mathbb{V}_{-\Delta}\left(\beta_{k}\right)$ for $j=1, \ldots, m$.

In the lemma below we characterise the operator $L_{\lambda A}$, given by the formula (2.8).

Lemma 2.1 For every $u \in \mathbb{H}$

$$
L_{\lambda A} u=\sum_{k=1}^{\infty} \sum_{j=1}^{m} \frac{1+\lambda \alpha_{j}}{1+\beta_{k}} \pi_{j}\left(u_{k}\right) \cdot f_{j}
$$

The proof of this lemma is standard, see for example the proof of Lemma 3.2 in [9].

Let us denote by $\sigma(L)$ the spectrum of a linear operator $L: \mathbb{H} \rightarrow \mathbb{H}$. From the above lemma it immediately follows the corollary:

Corollary 2.2 Let $L_{\lambda A}$ be defined by (2.8). Then:

$$
\sigma\left(L_{\lambda A}\right)=\left\{\frac{1+\lambda \alpha_{j}}{1+\beta_{k}}: \alpha_{j} \in \sigma(A), \beta_{k} \in \sigma\left(-\Delta ; B^{N}\right)\right\} .
$$

Moreover,

$$
\sigma\left(I d-L_{\lambda A}\right)=\left\{\frac{\beta_{k}-\lambda \alpha_{j}}{1+\beta_{k}}: \alpha_{j} \in \sigma(A), \beta_{k} \in \sigma\left(-\Delta ; B^{N}\right)\right\}
$$

Fix eigenvalues $\alpha_{j_{0}} \in \sigma(A)$ and $\beta_{k_{0}} \in \sigma\left(-\Delta ; B^{N}\right)$. Let $\mathbb{V}_{A}\left(\alpha_{j_{0}}\right)$ be the eigenspace associated with the eigenvalue $\alpha_{j_{0}}$ and $\mu_{A}\left(\alpha_{j_{0}}\right)=\operatorname{dim} \mathbb{V}_{A}\left(\alpha_{j_{0}}\right)$. Let $\Pi_{j_{0}}: \mathbb{R}^{m} \rightarrow \mathbb{R}^{m}$ 
be the orthogonal projection such that $\Pi_{j_{0}}\left(\mathbb{R}^{m}\right)=\mathbb{V}_{A}\left(\alpha_{j_{0}}\right)$ and define $\tilde{\Pi}_{j_{0}}: \mathbb{H} \rightarrow \mathbb{H}$ by $\left(\tilde{\Pi}_{j_{0}}(u)\right)(x)=\Pi_{j_{0}}(u(x))$. Denote

$$
\mathbb{V}_{-\Delta}\left(\beta_{k_{0}}\right)^{\mu_{A}\left(\alpha_{j_{0}}\right)}=\tilde{\Pi}_{j_{0}}\left(\bigoplus_{j=1}^{m} \mathbb{V}_{-\Delta}\left(\beta_{k_{0}}\right)\right) .
$$

It follows that

$$
\mathbb{V}_{-\Delta}\left(\beta_{k_{0}}\right)^{\mu_{A}\left(\alpha_{j_{0}}\right)}=\operatorname{span}\left\{h \cdot f: h \in \mathbb{V}_{-\Delta}\left(\beta_{k_{0}}\right), f \in \mathbb{V}_{A}\left(\alpha_{j_{0}}\right)\right\} \subset \mathbb{H}
$$

From Lemma 2.1 we obtain:

Corollary 2.3 If $\sigma(\lambda A) \cap \sigma\left(-\Delta ; B^{N}\right)=\left\{\alpha_{j_{1}}, \ldots, \alpha_{j_{s}}\right\}$, then

$$
\operatorname{ker}\left(I d-L_{\lambda A}\right)=\mathbb{V}_{-\Delta}\left(\alpha_{j_{1}}\right)^{\mu_{\lambda A}\left(\alpha_{j_{1}}\right)} \oplus \cdots \oplus \mathbb{V}_{-\Delta}\left(\alpha_{j_{s}}\right)^{\mu_{\lambda A}\left(\alpha_{j_{s}}\right)} .
$$

\subsection{The notion of bifurcation from the critical orbit}

Fix $u_{0} \in(\nabla F)^{-1}(0)$. Since $F$ is $\Gamma$-invariant, and therefore $\nabla F$ is $\Gamma$-equivariant, $\gamma u_{0} \in$ $(\nabla F)^{-1}(0)$ for all $\gamma \in \Gamma$, i.e. $\Gamma\left(u_{0}\right) \subset(\nabla F)^{-1}(0)$. We call such a set a critical orbit of $F$.

Note that $T_{u_{0}} \Gamma\left(u_{0}\right) \subset \operatorname{ker} \nabla^{2} F\left(u_{0}\right)$ and therefore $\operatorname{dim} \operatorname{ker} \nabla^{2} F\left(u_{0}\right) \geq \operatorname{dim} T_{u_{0}} \Gamma\left(u_{0}\right)=$ $\operatorname{dim} \Gamma\left(u_{0}\right)$. We assume that in this inequality there holds:

$$
\operatorname{dim} \operatorname{ker} \nabla^{2} F\left(u_{0}\right)=\operatorname{dim} \Gamma\left(u_{0}\right) .
$$

We call such an orbit non-degenerate.

By the equivariant Morse lemma, see [31], from (2.9) we conclude that $\Gamma\left(u_{0}\right)$ is isolated in $(\nabla F)^{-1}(0)$.

Since $u_{0} \in(\nabla F)^{-1}(0)$, the constant function $\tilde{u}_{0} \equiv u_{0}$ is a solution of the problem (2.1) for all $\lambda \in \mathbb{R}$. Therefore, $\left(\tilde{u}_{0}, \lambda\right)$, and consequently $\left(\gamma \tilde{u}_{0}, \lambda\right)$ for every $\gamma \in \Gamma$, is a critical point of the functional $\Phi$ given by (2.4). Since from (2.3) we have $\mathcal{G}\left(\tilde{u}_{0}\right)=\Gamma\left(\tilde{u}_{0}\right)$, we obtain a critical orbit of $\Phi$ and therefore a $\mathcal{G}$-orbit of weak solutions of (2.1) for all $\lambda \in \mathbb{R}$. Hence we can consider a family of solutions $\mathcal{T}=\mathcal{G}\left(\tilde{u}_{0}\right) \times \mathbb{R} \subset \mathbb{H} \times \mathbb{R}$. We call the elements of $\mathcal{T}$ the trivial solutions of (2.1). Put $\mathcal{N}=\left\{(v, \lambda) \in(\mathbb{H} \times \mathbb{R}) \backslash \mathcal{T}: \nabla_{v} \Phi(v, \lambda)=0\right\}$.

Definition 2.4 A local bifurcation from the orbit $\mathcal{G}\left(\tilde{u}_{0}\right) \times\left\{\lambda_{0}\right\} \subset \mathcal{T}$ of solutions of (2.1) occurs if the point $\left(\tilde{u}_{0}, \lambda_{0}\right)$ is an accumulation point of the set $\mathcal{N}$.

Remark 2.5 Note that if $\left(\tilde{u}_{0}, \lambda_{0}\right)$ is an accumulation point of $\mathcal{N}$ then for all $g \in \mathcal{G},\left(g \tilde{u}_{0}, \lambda_{0}\right)$ is also an accumulation point, since $\mathbb{H}$ is an orthogonal representation of $\mathcal{G}$. Therefore $\mathcal{G}\left(\tilde{u}_{0}\right) \subset$ $\operatorname{cl}(\mathcal{N})$.

Definition 2.6 A global bifurcation from the orbit $\mathcal{G}\left(\tilde{u}_{0}\right) \times\left\{\lambda_{0}\right\} \subset \mathcal{T}$ of solutions of (2.1) occurs if there is a connected component $\mathcal{C}\left(\lambda_{0}\right)$ of $\operatorname{cl}(\mathcal{N})$, containing $\mathcal{G}\left(\tilde{u}_{0}\right) \times\left\{\lambda_{0}\right\}$, such that either $\mathcal{C}\left(\lambda_{0}\right) \cap\left(\mathcal{T} \backslash\left(\mathcal{G}\left(\tilde{u}_{0}\right) \times\left\{\lambda_{0}\right\}\right)\right) \neq \emptyset$ or $\mathcal{C}\left(\lambda_{0}\right)$ is unbounded.

The set of all $\lambda_{0} \in \mathbb{R}$ such that a local (respectively global) bifurcation from the orbit $\mathcal{G}\left(\tilde{u}_{0}\right) \times\left\{\lambda_{0}\right\}$ occurs we denote by $B I F$ (respectively $G L O B$ ). Note that directly from the above definitions it follows that $G L O B \subset B I F$. 


\subsection{Admissible pair}

The notion of an admissible pair has been introduced in [18].

Fix a compact Lie group $G$ and let $H \in \overline{\operatorname{sub}}(G)$. Denote by $(H)_{G}$ the conjugacy class of $H$.

Definition 2.7 A pair $(G, H)$ is called admissible, if for any $K_{1}, K_{2} \in \overline{\operatorname{sub}}(H)$ the following condition is satisfied: if $\left(K_{1}\right)_{H} \neq\left(K_{2}\right)_{H}$, then $\left(K_{1}\right)_{G} \neq\left(K_{2}\right)_{G}$.

Lemma 2.8 The pair $(\Gamma \times S O(N),\{e\} \times S O(N))$ is admissible.

Proof Let us denote by $\mathrm{H}$ the group $\{e\} \times S O(N)$ and recall that $\mathcal{G}=\Gamma \times S O(N)$. Moreover, let $\tilde{K}_{1}, \tilde{K}_{2} \in \overline{\operatorname{sub}}(H)$. By definition of $H$ there are $K_{1}, K_{2} \in \overline{\operatorname{sub}}(S O(N))$ such that $\tilde{K}_{1}=$ $\{e\} \times K_{1}$ and $\tilde{K}_{2}=\{e\} \times K_{2}$. Suppose that $\left(\tilde{K}_{1}\right)_{\mathcal{G}}=\left(\tilde{K}_{2}\right)_{\mathcal{G}}$, i.e. $\left(\{e\} \times K_{1}\right)_{\mathcal{G}}=\left(\{e\} \times K_{2}\right)_{\mathcal{G}}$. Therefore there exists $(\gamma, \alpha) \in \mathcal{G}$ such that $\{e\} \times K_{1}=(\gamma, \alpha)\left(\{e\} \times K_{2}\right)(\gamma, \alpha)^{-1}$ and hence

$$
\begin{aligned}
\{e\} \times K_{1} & =\left\{\gamma e \gamma^{-1}\right\} \times \alpha K_{2} \alpha^{-1}=\{e\} \times \alpha K_{2} \alpha^{-1} \\
& =(e, \alpha)\left(\{e\} \times K_{2}\right)(e, \alpha)^{-1} .
\end{aligned}
$$

Thus $\left(\tilde{K}_{1}\right)_{H}=\left(\tilde{K}_{2}\right)_{H}$ and the proof is complete.

\section{Main results}

Consider the nonlinear system (2.1) with a potential $F$ satisfying (B1), (B2). Fix $u_{0} \in$ $(\nabla F)^{-1}(0)$ such that the orbit $\Gamma\left(u_{0}\right)$ is non-degenerate. We make two additional assumptions:

(B3) $F(u)=\frac{1}{2}(A u, u)-\left(A u_{0}, u\right)+g\left(u-u_{0}\right)$, where $A$ is a real symmetric $(m \times m)$-matrix and $\nabla g(u)=o(|u|)$ for $|u| \rightarrow 0$,

(B4) $\Gamma_{u_{0}}=\{e\}$.

From the assumption (B3) we conclude that the gradient of the functional associated with the Eq. (2.1) has the following form:

$$
\nabla_{u} \Phi(u, \lambda)=u-\tilde{u}_{0}-L_{\lambda A}\left(u-\tilde{u}_{0}\right)+\lambda \nabla \eta\left(u-\tilde{u}_{0}\right),
$$

where $L_{\lambda_{A}}: \mathbb{H} \rightarrow \mathbb{H}$ is an $S O(N)$-equivariant operator given by (2.8). Moreover, $\nabla \eta$ : $\mathbb{H} \rightarrow \mathbb{H}$ given by $\langle\nabla \eta(u), v\rangle_{\mathbb{H}}=\int_{B^{N}}(\nabla g(u(x)), v(x)) d x$ is an $S O(N)$-equivariant operator such that $\nabla \eta(u)=o\left(\|u\|_{\mathbb{H}}\right)$ for $\|u\|_{\mathbb{H}} \rightarrow 0$.

From the assumption (B4) it follows that $\mathcal{G}_{\tilde{u}_{0}}=\{e\} \times S O(N)$.

\subsection{Bifurcation from the critical orbit}

Following the standard notation we denote the linear part of $\nabla_{u} \Phi(\cdot, \lambda)$ at $\tilde{u}_{0}$ by $\nabla_{u}^{2} \Phi\left(\tilde{u}_{0}, \lambda\right) u$, thus $\nabla_{u}^{2} \Phi\left(\tilde{u}_{0}, \lambda\right) u=u-L_{\lambda A} u$.

Let us denote by $\Lambda$ the set $\bigcup_{\alpha_{j} \in \sigma(A) \backslash\{0\}} \bigcup_{\beta_{k} \in \sigma\left(-\Delta ; B^{N}\right)}\left\{\frac{\beta_{k}}{\alpha_{j}}\right\}$.

Lemma 3.1 If $\lambda_{0} \in B I F$, then $\lambda_{0} \in \Lambda$.

Proof We first observe that for all $\lambda \in \mathbb{R}$, since $\mathcal{G}\left(\tilde{u}_{0}\right)$ is a critical orbit of $\Phi(\cdot, \lambda)$, we have $\operatorname{dim} \operatorname{ker} \nabla_{u}^{2} \Phi\left(\tilde{u}_{0}, \lambda\right) \geq \operatorname{dim}\left(\mathcal{G}\left(\tilde{u}_{0}\right) \times\{\lambda\}\right)$.

Moreover if $\lambda_{0} \in B I F$, this inequality is strict. Indeed, if $\operatorname{dim} \operatorname{ker} \nabla_{u}^{2} \Phi\left(\tilde{u}_{0}, \lambda_{0}\right)=$ $\operatorname{dim}\left(\mathcal{G}\left(\tilde{u}_{0}\right) \times\left\{\lambda_{0}\right\}\right)$, then by the equivariant implicit function theorem (see Theorem 5.1) 
there exists $\varepsilon>0$ such that the only solutions of the equation $\nabla_{u} \Phi(u, \lambda)=0$ are elements of $\mathcal{G}\left(\tilde{u}_{0}\right) \times\{\lambda\}$ for $\lambda \in\left(\lambda_{0}-\varepsilon, \lambda_{0}+\varepsilon\right)$. From this we obtain $\lambda_{0} \notin B I F$. Therefore, if $\lambda_{0} \in B I F$,

$$
\operatorname{dim} \operatorname{ker} \nabla_{u}^{2} \Phi\left(\tilde{u}_{0}, \lambda_{0}\right)>\operatorname{dim}\left(\mathcal{G}\left(\tilde{u}_{0}\right) \times\left\{\lambda_{0}\right\}\right) .
$$

Since $\mathcal{G}\left(\tilde{u}_{0}\right)=\Gamma\left(\tilde{u}_{0}\right)$, we conclude from (2.9) and (3.1) that $\operatorname{dim} \operatorname{ker} \nabla_{u}^{2} \Phi\left(\tilde{u}_{0}, \lambda_{0}\right)>$ $\operatorname{dim}\left(\mathcal{G}\left(\tilde{u}_{0}\right) \times\left\{\lambda_{0}\right\}\right)=\operatorname{dim} \operatorname{ker} \nabla^{2} F\left(u_{0}\right)$, i.e. $\operatorname{dim} \operatorname{ker}\left(I d-L_{\lambda_{0} A}\right)>\operatorname{dim} \operatorname{ker} A$. Using Corollary 2.2 we obtain that this condition is satisfied if and only if $\left\{\left(\alpha_{j}, \beta_{k}\right) \in \sigma(A) \times\right.$ $\left.\sigma\left(-\Delta ; B^{N}\right): \beta_{k}=\lambda_{0} \alpha_{j}\right\} \neq\{(0,0)\}$. Therefore there are $\left(\alpha_{j}, \beta_{k}\right) \in \sigma(A) \backslash\{0\} \times$ $\sigma\left(-\Delta, B^{N}\right)$ such that $\beta_{k}=\lambda_{0} \cdot \alpha_{j}$, i.e. $\lambda_{0} \in \Lambda$.

Fix $\lambda_{0} \in \Lambda$ and choose $\varepsilon>0$ such that $\Lambda \cap\left[\lambda_{0}-\varepsilon, \lambda_{0}+\varepsilon\right]=\left\{\lambda_{0}\right\}$. From the definition of $\Lambda$ such a choice is always possible.

Since $\lambda_{0} \pm \varepsilon \notin \Lambda$, Lemma 3.1 implies that $\lambda_{0} \pm \varepsilon \notin B I F$ and therefore $\mathcal{G}\left(\tilde{u}_{0}\right) \subset \mathbb{H}$ is an isolated critical orbit of the $\mathcal{G}$-invariant functionals $\Phi\left(\cdot, \lambda_{0} \pm \varepsilon\right): \mathbb{H} \rightarrow \mathbb{R}$. From this and the properties of flows induced by gradient operators, we conclude that $\mathcal{G}\left(\tilde{u}_{0}\right)$ is also an isolated invariant set (in the sense of the equivariant Conley index theory, see [13]) for the flows induced by the operators $-\nabla_{u} \Phi\left(\cdot, \lambda_{0} \pm \varepsilon\right)$. Therefore, the indices $\mathcal{C} \mathcal{I}_{\mathcal{G}}\left(\mathcal{G}\left(\tilde{u}_{0}\right),-\nabla_{u} \Phi\left(\cdot, \lambda_{0}\right.\right.$ $-\varepsilon)), \mathcal{C} \mathcal{I}_{\mathcal{G}}\left(\mathcal{G}\left(\tilde{u}_{0}\right),-\nabla_{u} \Phi\left(\cdot, \lambda_{0}+\varepsilon\right)\right)$ are well-defined. In the following we study when they are not equal.

Assume that $\sigma\left(\lambda_{0} A\right) \cap \sigma\left(-\Delta ; B^{N}\right)=\left\{\alpha_{j_{1}}, \ldots, \alpha_{j_{s}}\right\}$. We consider the conditions:

(C1) $\lambda_{0} \neq 0$ and there is $i \in\{1, \ldots, s\}$ satisfying $\operatorname{dim} \mathbb{V}_{-\Delta}\left(\alpha_{j_{i}}\right)>1$,

(C2) $\lambda_{0} \neq 0, \operatorname{dim} \mathbb{V}_{-\Delta}\left(\alpha_{j_{i}}\right)=1$ for every $i \in\{1, \ldots, s\}$ and $\operatorname{dim} \operatorname{ker}\left(I d-L_{\lambda_{0} A}\right)-$ $\operatorname{dim} \operatorname{ker} A$ is an odd number,

(C3) $\lambda_{0}=0$ and $\sum_{\alpha \in \sigma_{+}(A)} \mu_{A}(\alpha)-\sum_{\alpha \in \sigma_{-}(A)} \mu_{A}(\alpha)$ is odd.

Remark 3.2 Note that we can reformulate conditions (C1)-(C3) in the following way:

(C1') $\lambda_{0} \neq 0$ and there is $i \in\{1, \ldots, s\}$ such that $\mathbb{V}_{-\Delta}\left(\alpha_{j_{i}}\right)$ is a nontrivial $S O(N)$ representation,

(C2') $\lambda_{0} \neq 0, \operatorname{dim} \mathbb{V}_{-\Delta}\left(\alpha_{j_{i}}\right)=1$ for every $i \in\{1, \ldots, s\}$ and $\sum_{i=1}^{s} \mu_{\lambda_{0} A}\left(\alpha_{j_{i}}\right)-\mu_{A}(0)$ is odd,

(C3') $\lambda_{0}=0$ and $m-\operatorname{dim} \operatorname{ker} A$ is odd.

Indeed,

(1) $\operatorname{dim} \mathbb{V}_{-\Delta}\left(\alpha_{j_{i}}\right)>1$ if and only if $\mathbb{V}_{-\Delta}\left(\alpha_{j_{i}}\right)$ is a nontrivial $S O(N)$-representation, see Remark 5.11;

(2) since $\operatorname{dim} \mathbb{V}_{-\Delta}\left(\alpha_{j_{i}}\right)=1$, from Corollary 2.3 we obtain $\operatorname{dim} \operatorname{ker}\left(I d-L_{\lambda_{0} A}\right)=$ $\sum_{i=1}^{s} \mu_{\lambda_{0} A}\left(\alpha_{j_{i}}\right)$

(3) since $\sum_{\alpha \in \sigma_{+}(A)} \mu_{A}(\alpha)+\sum_{\alpha \in \sigma_{-}(A)} \mu_{A}(\alpha)+\mu_{A}(0)=m$, if $m-\operatorname{dim} \operatorname{ker} A$ is odd, then so is $\sum_{\alpha \in \sigma_{+}(A)} \mu_{A}(\alpha)-\sum_{\alpha \in \sigma_{-}(A)} \mu_{A}(\alpha)$.

Theorem 3.3 Assume that $\lambda_{0} \in \Lambda$ and one of the conditions (C1)-(C3) is satisfied. Then

$$
\mathcal{C} \mathcal{I}_{\mathcal{G}}\left(\mathcal{G}\left(\tilde{u}_{0}\right),-\nabla_{u} \Phi\left(\cdot, \lambda_{0}-\varepsilon\right)\right) \neq \mathcal{C} \mathcal{I}_{\mathcal{G}}\left(\mathcal{G}\left(\tilde{u}_{0}\right),-\nabla_{u} \Phi\left(\cdot, \lambda_{0}+\varepsilon\right)\right) .
$$

Proof Denote by $\tilde{\mathbb{H}} \subset \mathbb{H}$ the linear subspace normal to $\mathcal{G}\left(\tilde{u}_{0}\right)$ at $\tilde{u}_{0}$, i.e. $\tilde{\mathbb{H}}=T_{\tilde{u}_{0}}^{\perp} \mathcal{G}\left(\tilde{u}_{0}\right) \subset$ $\mathbb{H}$. We start the proof with showing that we can reduce comparing the Conley indices $\mathcal{C} \mathcal{I}_{\mathcal{G}}\left(\mathcal{G}\left(\tilde{u}_{0}\right),-\nabla_{u} \Phi\left(\cdot, \lambda_{0} \pm \varepsilon\right)\right)$ to comparing Euler characteristics of some indices on the space $\tilde{\mathbb{H}}$. 
For $n \geq 1$ put $\mathbb{H}^{n}=\bigoplus_{k=1}^{n} \mathbb{H}_{k}$ and

$$
\Phi^{n}=\Phi_{\mid \mathbb{H}^{n} \times \mathbb{R}}: \mathbb{H}^{n} \times \mathbb{R} \rightarrow \mathbb{R} .
$$

Note that $\mathcal{G}\left(\tilde{u}_{0}\right)=\Gamma\left(\tilde{u}_{0}\right) \subset T_{\tilde{u}_{0}} \Gamma\left(\tilde{u}_{0}\right) \oplus T_{\tilde{u}_{0}}^{\perp} \Gamma\left(\tilde{u}_{0}\right) \approx \mathbb{R}^{m} \approx \mathbb{H}_{1}$ (by $T_{\tilde{u}_{0}}^{\perp} \Gamma\left(\tilde{u}_{0}\right)$ we understand the complement of the space $T_{\tilde{u}_{0}} \Gamma\left(\tilde{u}_{0}\right)$ in $\left.\mathbb{H}_{1}\right)$. Therefore $\mathcal{G}\left(\tilde{u}_{0}\right)$ is a critical orbit of $\Phi^{n}\left(\cdot, \lambda_{0} \pm\right.$ $\varepsilon$ ) for $n \geq 1$. Note that, from the choice of $\varepsilon$ and the definition of $\Phi^{n}$, this orbit is nondegenerate.

Since $\nabla_{u} \Phi(\cdot, \lambda)$ is a completely continuous perturbation of the identity for all $\lambda \in \mathbb{R}$, from the definition of the infinite dimensional equivariant Conley index, see Sect. 5.2, the assertion of the theorem is equivalent to

$$
C I_{\mathcal{G}}\left(\mathcal{G}\left(\tilde{u}_{0}\right),-\nabla_{u} \Phi^{n}\left(\cdot, \lambda_{0}-\varepsilon\right)\right) \neq C I_{\mathcal{G}}\left(\mathcal{G}\left(\tilde{u}_{0}\right),-\nabla_{u} \Phi^{n}\left(\cdot, \lambda_{0}+\varepsilon\right)\right)
$$

for $n$ sufficiently large. Obviously, the above inequality is implied by

$$
\chi_{\mathcal{G}}\left(C I_{\mathcal{G}}\left(\mathcal{G}\left(\tilde{u}_{0}\right),-\nabla_{u} \Phi^{n}\left(\cdot, \lambda_{0}-\varepsilon\right)\right)\right) \neq \chi_{\mathcal{G}}\left(C I_{\mathcal{G}}\left(\mathcal{G}\left(\tilde{u}_{0}\right),-\nabla_{u} \Phi^{n}\left(\cdot, \lambda_{0}+\varepsilon\right)\right)\right) .
$$

It is known that the $\mathcal{G}$-action on $\mathbb{H}$ given by (2.3) defines a $\mathcal{G}_{\tilde{u}_{0}}$-action on $\tilde{\mathbb{H}}$. Recall that $\mathcal{G}_{\tilde{u}_{0}}=\{e\} \times S O(N)$. Hence $\tilde{\mathbb{H}}$ is an orthogonal $S O(N)$-representation.

For $n \geq 1$ put $\tilde{\mathbb{H}}^{n}=\mathbb{H}^{n} \cap \tilde{\mathbb{H}}=T_{\tilde{u}_{0}}^{\perp} \Gamma\left(\tilde{u}_{0}\right) \oplus \bigoplus_{k=2}^{n} \mathbb{H}_{k}$ and define $\Psi_{ \pm}^{n}=\Phi^{n}\left(\cdot, \lambda_{0} \pm\right.$ $\varepsilon)_{\mid \tilde{\mathbb{H}}^{n}}: \tilde{\mathbb{H}}^{n} \rightarrow \mathbb{R}$. From this definition the functionals $\Psi_{ \pm}^{n}$ are $S O(N)$-invariant. Since $\mathcal{G}\left(\tilde{u}_{0}\right)$ is a non-degenerate critical orbit of $\Phi^{n}\left(\cdot, \lambda_{0} \pm \varepsilon\right), \tilde{u}_{0} \in \tilde{\mathbb{H}}$ is a non-degenerate critical point of $\Psi_{ \pm}^{n}$. Hence $\left\{\tilde{u}_{0}\right\}$ is an isolated invariant set (in the sense of the Conley index theory) of the flows generated by $-\nabla \Psi_{ \pm}^{n}$.

Note that since $\mathcal{G}_{\tilde{u}_{0}}=\{e\} \times S O(N)$, by Lemma 2.8 the pair $\left(\mathcal{G}, \mathcal{G}_{\tilde{u}_{0}}\right)$ is admissible. Therefore, using Fact 5.6 we obtain that the assertion reduces to

$$
\chi_{\mathcal{G}_{\tilde{u}_{0}}}\left(C I_{\mathcal{G}_{\tilde{u}_{0}}}\left(\left\{\tilde{u}_{0}\right\},-\nabla \Psi_{-}^{n}\right)\right) \neq \chi_{\mathcal{G}_{\tilde{u}_{0}}}\left(C I_{\mathcal{G}_{\tilde{u}_{0}}}\left(\left\{\tilde{u}_{0}\right\},-\nabla \Psi_{+}^{n}\right)\right)
$$

for $n \in \mathbb{N}$ sufficiently large. It is easy to see that this inequality is equivalent to

$$
\chi_{S O(N)}\left(C I_{S O(N)}\left(\left\{\tilde{u}_{0}\right\},-\nabla \Psi_{-}^{n}\right)\right) \neq \chi_{S O(N)}\left(C I_{S O(N)}\left(\left\{\tilde{u}_{0}\right\},-\nabla \Psi_{+}^{n}\right)\right) .
$$

We proceed to show that there exists $n_{0} \in \mathbb{N}$ such that for $n \geq n_{0}$

$$
C I_{S O(N)}\left(\left\{\tilde{u}_{0}\right\},-\nabla \Psi_{ \pm}^{n}\right)=C I_{S O(N)}\left(\left\{\tilde{u}_{0}\right\},-\nabla \Psi_{ \pm}^{n_{0}}\right) .
$$

Let $v \in \mathbb{N}$. For $\delta>0$ sufficiently small and $\lambda \in\left[\lambda_{0}-\varepsilon, \lambda_{0}+\varepsilon\right]$ we define $S O(N)$-equivariant gradient homotopy $H_{\lambda}^{v}:\left(D_{\delta}\left(\tilde{u}_{0}, \tilde{\mathbb{H}}^{v}\right) \times[0,1], \partial D_{\delta}\left(\tilde{u}_{0}, \tilde{\mathbb{H}}^{v}\right) \times[0,1]\right) \rightarrow$ $\left(\tilde{\mathbb{H}}^{v}, \tilde{\mathbb{H}}^{v} \backslash\{0\}\right)$ by

$$
H_{\lambda}^{v}(u, t)=u-\tilde{u}_{0}-L_{\lambda A}\left(u-\tilde{u}_{0}\right)+t \lambda_{0} P_{\nu} \circ \nabla \eta\left(u-\tilde{u}_{0}\right),
$$

where $P_{\nu}: \tilde{\mathbb{H}} \rightarrow \tilde{\mathbb{H}}^{v}$ is the orthogonal $S O(N)$-equivariant projection onto $\tilde{\mathbb{H}}{ }^{v}$. Note that from Lemma 2.1 we have $P_{\nu} \circ L_{\lambda A}=L_{\lambda A} \circ P_{\nu}$ and hence this homotopy is well-defined.

Let us denote by $\xi_{\lambda}^{\nu}: \tilde{\mathbb{H}}^{\nu} \rightarrow \mathbb{R}$ the $S O(N)$-invariant potential of $H_{\lambda}^{v}(\cdot, 0)$. It is clear that $\nabla \xi_{\lambda}^{v}: \tilde{\mathbb{H}}^{v} \rightarrow \tilde{\mathbb{H}}^{v}$ is a self-adjoint $S O(N)$-equivariant linear map and is given by the formula $\nabla \xi_{\lambda}^{v}=\left(I d-L_{\lambda A}\right)_{\mid \tilde{H}^{\nu}}$. From the homotopy invariance of the Conley index, see Theorem 5.3, we obtain

$$
C I_{S O(N)}\left(\left\{\tilde{u}_{0}\right\},-\nabla \Psi_{ \pm}^{\nu}\right)=C I_{S O(N)}\left(\left\{\tilde{u}_{0}\right\},-\nabla \xi_{\lambda_{0} \pm \varepsilon}^{\nu}\right) .
$$


Recall that $\left(\beta_{k}\right)$ denotes the sequence of the eigenvalues of the Neumann Laplacian and note that $\beta_{k} \rightarrow+\infty$. Therefore, there exists $n_{0} \in \mathbb{N}$ such that the inequalities $\frac{\beta_{n}-\left(\lambda_{0} \pm \varepsilon\right) \alpha_{j}}{1+\beta_{n}}$ $>0$ hold for every $n \geq n_{0}$ and $\alpha_{j} \in \sigma(A)$. Hence, by Corollary 2.2, there exists $n_{0} \in \mathbb{N}$ such that $m^{-}\left(\nabla \xi_{\lambda_{0} \pm \varepsilon}^{n}\right)=m^{-}\left(\nabla \xi_{\lambda_{0} \pm \varepsilon}^{n_{0}}\right)$ for every $n \geq n_{0}$, where $m^{-}(\cdot)$ is the Morse index. Since $\left(\nabla \xi_{\lambda_{0} \pm \varepsilon}^{n}\right)_{\mid \tilde{H}^{n_{0}}}=\nabla \xi_{\lambda_{0} \pm \varepsilon}^{n_{0}}$, the eigenspaces corresponding to the negative eigenvalues of $\nabla \xi_{\lambda_{0} \pm \varepsilon}^{n}$ and $\nabla \xi_{\lambda_{0} \pm \varepsilon}^{n_{0}}$ are the same $S O(N)$-representations. Thus, from Theorem 5.2,

$$
C I_{S O(N)}\left(\left\{\tilde{u}_{0}\right\},-\nabla \xi_{\lambda_{0} \pm \varepsilon}^{n}\right)=C I_{S O(N)}\left(\left\{\tilde{u}_{0}\right\},-\nabla \xi_{\lambda_{0} \pm \varepsilon}^{n_{0}}\right),
$$

which implies (3.5).

To finish the proof of (3.4), and therefore also of the assertion, we will show that

$$
\chi_{S O(N)}\left(C I_{S O(N)}\left(\left\{\tilde{u}_{0}\right\},-\nabla \Psi_{+}^{n_{0}}\right)\right) \neq \chi_{S O(N)}\left(C I_{S O(N)}\left(\left\{\tilde{u}_{0}\right\},-\nabla \Psi_{-}^{n_{0}}\right)\right) .
$$

Denote by $\mathcal{W}(\lambda)$ the direct sum of the eigenspaces of $I d-L_{\lambda A}$ (i.e. of $\nabla \xi_{\lambda}^{n_{0}}$ ) corresponding to the negative eigenvalues and by $\mathcal{V}(\lambda)$ the eigenspace corresponding to the zero eigenvalue. Note that from Corollary 2.2,

$$
\begin{gathered}
\mathcal{W}(\lambda)=\left(\bigoplus_{\alpha_{j} \in \sigma(A)} \bigoplus_{\substack{\beta_{k} \in \sigma\left(-\Delta ; B^{N}\right) \\
\beta_{k}<\lambda \alpha_{j}}} \mathbb{V}_{-\Delta}\left(\beta_{k}\right)^{\mu_{A}\left(\alpha_{j}\right)}\right) \cap \tilde{\mathbb{H}}, \\
\mathcal{V}(\lambda)=\left(\bigoplus_{\alpha_{j} \in \sigma(A)} \bigoplus_{\substack{\beta_{k} \in \sigma\left(-\Delta ; B^{N}\right) \\
\beta_{k}=\lambda \alpha_{j}}} \mathbb{V}_{-\Delta}\left(\beta_{k}\right)^{\mu_{A}\left(\alpha_{j}\right)}\right) \cap \tilde{\mathbb{H}} .
\end{gathered}
$$

From Theorem 5.2, $C I_{S O(N)}\left(\left\{\tilde{u}_{0}\right\},-\nabla \xi_{\lambda_{0} \pm \varepsilon}^{n_{0}}\right)$ are $S O(N)$-homotopy types of $S^{\mathcal{W}\left(\lambda_{0} \pm \varepsilon\right)}$. Hence, from (3.6),

$$
\chi_{S O(N)}\left(C I_{S O(N)}\left(\left\{\tilde{u}_{0}\right\},-\nabla \Psi_{ \pm}^{n_{0}}\right)\right)=\chi_{S O(N)}\left(S^{\mathcal{W}\left(\lambda_{0} \pm \varepsilon\right)}\right) .
$$

(1) Suppose that $\lambda_{0}>0$ and $\varepsilon$ is such that $\lambda_{0}-\varepsilon>0$. Recall that $\beta_{k} \geq 0$ for all $\beta_{k} \in$ $\sigma\left(-\Delta ; B^{N}\right)$. Then $\mathcal{W}\left(\lambda_{0}+\varepsilon\right)=\mathcal{W}\left(\lambda_{0}-\varepsilon\right) \oplus \mathcal{V}\left(\lambda_{0}\right)$. If the assumption $(\mathrm{C} 1)$ is satisfied, then, by Theorem 5.4 and Remark 5.11, we obtain $\chi_{S O(N)}\left(S^{\mathcal{V}\left(\lambda_{0}\right)}\right) \neq \mathbb{I} \in U(S O(N))$. Similarly, if (C2) is fulfilled, then $\mathcal{V}\left(\lambda_{0}\right)$ is a trivial $S O(N)$-representation and, from Corollary 2.2 and the definition of $\tilde{\mathbb{H}}, \operatorname{dim} \mathcal{V}\left(\lambda_{0}\right)=\operatorname{dim} \operatorname{ker}\left(I d-L_{\lambda_{0} A}\right)-\operatorname{dim} \operatorname{ker} A$ is odd. Therefore:

$$
\chi_{S O(N)}\left(S^{\mathcal{V}\left(\lambda_{0}\right)}\right)=(-1)^{\operatorname{dim} \mathcal{V}\left(\lambda_{0}\right)} \chi_{S O(N)}\left(S O(N) / S O(N)^{+}\right)=-\mathbb{I} .
$$

In both cases we have

$$
\begin{aligned}
& \chi_{S O(N)}\left(C I_{S O(N)}\left(\left\{\tilde{u}_{0}\right\},-\nabla \Psi_{+}^{n_{0}}\right)\right) \\
& \quad=\chi_{S O(N)}\left(S^{\mathcal{W}\left(\lambda_{0}-\varepsilon\right) \oplus \mathcal{V}\left(\lambda_{0}\right)}\right) \\
& =\chi_{S O(N)}\left(S^{\mathcal{W}\left(\lambda_{0}-\varepsilon\right)}\right) \star \chi_{S O(N)}\left(S^{\mathcal{V}\left(\lambda_{0}\right)}\right) \\
& \quad \neq \chi_{S O(N)}\left(S^{\mathcal{W}\left(\lambda_{0}-\varepsilon\right)}\right) \\
& =\chi_{S O(N)}\left(C I_{S O(N)}\left(\left\{\tilde{u}_{0}\right\},-\nabla \Psi_{-}^{n_{0}}\right)\right) .
\end{aligned}
$$

In the second equality we use the fact that $S^{\mathcal{W}\left(\lambda_{0}-\varepsilon\right) \oplus \mathcal{V}\left(\lambda_{0}\right)}$ is $S O(N)$-homeomorphic to $S^{\mathcal{W}\left(\lambda_{0}-\varepsilon\right)} \wedge S^{\mathcal{V}\left(\lambda_{0}\right)}$ and the formula for multiplication in $U(S O(N))$, see (5.1). Then we use invertibility of $\chi_{S O(N)}\left(S^{\mathcal{W}\left(\lambda_{0}-\varepsilon\right)}\right)$ in $U(S O(N))$, see [10]. 
(2) Suppose that $\lambda_{0}<0$. Then $\mathcal{W}\left(\lambda_{0}-\varepsilon\right)=\mathcal{W}\left(\lambda_{0}+\varepsilon\right) \oplus \mathcal{V}\left(\lambda_{0}\right)$ and hence

$$
\chi_{S O(N)}\left(C I_{S O(N)}\left(\left\{\tilde{u}_{0}\right\},-\nabla \Psi_{+}^{n_{0}}\right)\right) \neq \chi_{S O(N)}\left(C I_{S O(N)}\left(\left\{\tilde{u}_{0}\right\},-\nabla \Psi_{-}^{n_{0}}\right)\right),
$$

as before.

(3) Finally, suppose that $\lambda_{0}=0$. Then, since

$$
\mathcal{W}( \pm \varepsilon)=\bigoplus_{\alpha_{j} \in \sigma_{ \pm}(A)} \mathbb{V}_{-\Delta}(0)^{\mu_{A}\left(\alpha_{j}\right)},
$$

and therefore $\mathcal{W}( \pm \varepsilon)$ are trivial $S O(N)$-representations,

$$
\begin{aligned}
& \chi_{S O(N)}\left(C I_{S O(N)}\left(\left\{\tilde{u}_{0}\right\},-\nabla \Psi_{ \pm}^{n_{0}}\right)\right) \\
& =\chi_{S O(N)}\left(S^{\mathcal{W}( \pm \varepsilon)}\right) \\
& =(-1)^{\operatorname{dim} \mathcal{W}( \pm \varepsilon)} \cdot \chi_{S O(N)}\left(S O(N) / S O(N)^{+}\right) \\
& =(-1)^{\operatorname{dim} \mathcal{W}( \pm \varepsilon)} \cdot \mathbb{I} .
\end{aligned}
$$

Hence, because the assumption (C3) implies that $\operatorname{dim} \mathcal{W}(\varepsilon)-\operatorname{dim} \mathcal{W}(-\varepsilon)$ is odd, we have

$$
\chi_{S O(N)}\left(C I_{S O(N)}\left(\left\{\tilde{u}_{0}\right\},-\nabla \Psi_{+}^{n_{0}}\right)\right) \neq \chi_{S O(N)}\left(C I_{S O(N)}\left(\left\{\tilde{u}_{0}\right\},-\nabla \Psi_{-}^{n_{0}}\right)\right),
$$

which completes the proof.

Remark 3.4 Note that in the proof of Theorem 3.3 we have obtained that there is in fact a change of the Euler characteristic of the Conley index at $\mathcal{G}\left(\tilde{u}_{0}\right) \times\left\{\lambda_{0}\right\}$. More precisely, we have the inequality (3.3), i.e.

$$
\chi_{\mathcal{G}}\left(C I_{\mathcal{G}}\left(\mathcal{G}\left(\tilde{u}_{0}\right),-\nabla_{u} \Phi^{n}\left(\cdot, \lambda_{0}-\varepsilon\right)\right)\right) \neq \chi_{\mathcal{G}}\left(C I_{\mathcal{G}}\left(\mathcal{G}\left(\tilde{u}_{0}\right),-\nabla_{u} \Phi^{n}\left(\cdot, \lambda_{0}+\varepsilon\right)\right)\right),
$$

where $\Phi^{n}$ is defined by (3.2) and $n$ is sufficiently large.

Now we are in a position to prove one of the main results of our paper, namely the global bifurcation theorem.

Theorem 3.5 Consider the system (2.1) with the potential $F$ and $u_{0} \in \nabla F^{-1}(0)$ satisfying assumptions (B1)-(B4). Assume that $\lambda_{0} \in \Lambda$ and one of the conditions $(C 1)-(C 3)$ is satisfied. Then a global bifurcation of solutions of (2.1) occurs from the orbit $\mathcal{G}\left(\tilde{u}_{0}\right) \times\left\{\lambda_{0}\right\}$.

Proof Throughout the proof we follow the notation of the proof of Theorem 3.3. Let $\mathcal{U} \subset \mathbb{H}$ be an open, bounded and $\mathcal{G}$-invariant subset such that $\nabla_{u} \Phi\left(\cdot, \lambda_{0} \pm \varepsilon\right)^{-1}(0) \cap \mathcal{U}=\mathcal{G}\left(\tilde{u}_{0}\right)$. From Theorem 5.7 it follows that to prove the assertion it is enough to show

$$
\nabla_{\mathcal{G}}-\operatorname{deg}\left(\nabla_{u} \Phi\left(\cdot, \lambda_{0}+\varepsilon\right), \mathcal{U}\right) \neq \nabla_{\mathcal{G}}-\operatorname{deg}\left(\nabla_{u} \Phi\left(\cdot, \lambda_{0}-\varepsilon\right), \mathcal{U}\right),
$$

where $\nabla_{\mathcal{G}}-\operatorname{deg}(\cdot, \cdot)$ is the degree for equivariant gradient maps, see Sect. 5.3. From the definition of the degree, see (5.2), it follows that

$$
\nabla_{\mathcal{G}}-\operatorname{deg}\left(\nabla_{u} \Phi\left(\cdot, \lambda_{0} \pm \varepsilon\right), \mathcal{U}\right)=\nabla_{\mathcal{G}}-\operatorname{deg}\left(\nabla_{u} \Phi^{n_{0}}\left(\cdot, \lambda_{0} \pm \varepsilon\right), \mathcal{U} \cap \mathbb{H}^{n_{0}}\right)
$$

for $n_{0}$ sufficiently large. Note that $\mathcal{G}\left(\tilde{u}_{0}\right) \subset \mathcal{U} \cap \mathbb{H}^{n_{0}}$ and hence, by Theorem 5.8, we obtain

$$
\nabla_{\mathcal{G}}-\operatorname{deg}\left(\nabla_{u} \Phi^{n_{0}}\left(\cdot, \lambda_{0} \pm \varepsilon\right), \mathcal{U} \cap \mathbb{H}^{n_{0}}\right)=\chi_{\mathcal{G}}\left(C I_{\mathcal{G}}\left(\mathcal{G}\left(\tilde{u}_{0}\right),-\nabla_{u} \Phi^{n_{0}}\left(\cdot, \lambda_{0} \pm \varepsilon\right)\right)\right)
$$

Therefore, the assertion follows from Remark 3.4. 
Since the global bifurcation implies the local one, as an immediate corollary of the above theorem we obtain the following:

Corollary 3.6 Consider the system (2.1) with the potential $F$ and $u_{0} \in \nabla F^{-1}(0)$ satisfying assumptions (B1)-(B4). Assume that $\lambda_{0} \in \Lambda$ and one of the conditions $(C 1)-(C 3)$ is satisfied. Then a local bifurcation of solutions of (2.1) occurs from the orbit $\mathcal{G}\left(\tilde{u}_{0}\right) \times\left\{\lambda_{0}\right\}$.

Remark 3.7 The above corollary can be obtained directly from Theorem 3.3. Indeed, from this theorem it follows that if one of the conditions (C1)-(C3) is satisfied then $\mathcal{C} \mathcal{I}_{\mathcal{G}}\left(\mathcal{G}\left(\tilde{u}_{0}\right),-\nabla_{u} \Phi\left(\cdot, \lambda_{0}-\varepsilon\right)\right) \neq \mathcal{C} \mathcal{I}_{\mathcal{G}}\left(\mathcal{G}\left(\tilde{u}_{0}\right),-\nabla_{u} \Phi\left(\cdot, \lambda_{0}+\varepsilon\right)\right)$, for sufficiently small $\varepsilon>0$. Following for example the idea of the proof of Theorem 2.1 of [26], using the continuation property of the Conley index, one can prove that the change of the Conley index implies a local bifurcation of critical orbits.

In Theorem 3.5 we have proved that if the assumption (C3) is satisfied, then $0 \in G L O B$. On the other hand, repeating the argument from the proof of this theorem it is easy to show that if the number $\sum_{\alpha_{j} \in \sigma_{+}(A)} \mu_{A}\left(\alpha_{j}\right)-\sum_{\alpha_{j} \in \sigma_{-}(A)} \mu_{A}\left(\alpha_{j}\right)$ is even, then the Euler characteristics $\chi_{\mathcal{G}}\left(C I_{\mathcal{G}}\left(\mathcal{G}\left(\tilde{u}_{0}\right),-\nabla_{u} \Phi^{n_{0}}(\cdot, \varepsilon)\right)\right.$ and $\chi_{\mathcal{G}}\left(C I_{\mathcal{G}}\left(\mathcal{G}\left(\tilde{u}_{0}\right),-\nabla_{u} \Phi^{n_{0}}(\cdot,-\varepsilon)\right)\right)$ are equal. Therefore, we do not know whether $0 \in G L O B$. However, under the assumption weaker than (C3) we can prove the result concerning the local bifurcation.

Theorem 3.8 Consider the system (2.1) with the potential $F$ and $u_{0} \in \nabla F^{-1}(0)$ satisfying assumptions (B1)-(B4). Assume that $\lambda_{0}=0$ and $\sum_{\alpha_{j} \in \sigma_{+}(A)} \mu_{A}\left(\alpha_{j}\right) \neq \sum_{\alpha_{j} \in \sigma_{-}(A)} \mu_{A}\left(\alpha_{j}\right)$. Then a local bifurcation of solutions of (2.1) occurs from the orbit $\mathcal{G}\left(\tilde{u}_{0}\right) \times\{0\}$.

Proof Using the notation of the proof of Theorem 3.3, we observe that $\mathcal{W}( \pm \varepsilon)$ are trivial $S O(N)$-representations. Therefore $C I_{S O(N)}\left(\left\{\tilde{u}_{0}\right\},-\nabla \Psi_{ \pm}^{n_{0}}\right)$ are $S O(N)$-homotopy types of $S^{\operatorname{dim} \mathcal{W}( \pm \varepsilon)}$. Using information from [18] (namely Theorem 3.1 and the equality (2.11)) and from [14] (Lemma 1.88) we obtain that $C I_{\mathcal{G}}\left(\mathcal{G}\left(\tilde{u}_{0}\right),-\nabla_{u} \Phi^{n_{0}}(\cdot, \pm \varepsilon)\right)$ are $\mathcal{G}$-homotopy types of

$$
\left(\mathcal{G} / \mathcal{G}_{\tilde{u}_{0}} \times S^{\operatorname{dim} \mathcal{W}( \pm \varepsilon)}\right) /\left(\mathcal{G} / \mathcal{G}_{\tilde{u}_{0}} \times\{*\}\right) .
$$

From Proposition 1.53 of [14], we obtain that the above is $\mathcal{G}$-homotopy equivalent to

$$
X_{ \pm}=\left(\mathcal{G}\left(\tilde{u}_{0}\right) \times S^{\operatorname{dim} \mathcal{W}( \pm \varepsilon)}\right) /\left(\mathcal{G}\left(\tilde{u}_{0}\right) \times\{*\}\right) .
$$

But $X_{+}$and $X_{-}$are different $\mathcal{G}$-homotopy types. Indeed, if $X_{+}$and $X_{-}$are the same $\mathcal{G}$ homotopy types, then the orbit spaces $X_{+} / \mathcal{G}$ and $X_{-} / \mathcal{G}$ are the same homotopy types. This is impossible, since the spaces $X_{ \pm} / \mathcal{G}$ are homotopy types of $S^{\operatorname{dim} \mathcal{W}( \pm \varepsilon)}$, see [29]. Similarly as in Remark 3.7 we use the fact, that the change of the Conley indices implies the local bifurcation. This shows the assertion.

\subsection{Symmetry breaking}

In this section we consider the symmetry breaking problem, i.e. the change of the isotropy groups of solutions of (2.1) along connected sets. More precisely, we characterise bifurcation orbits of the Eq. (2.1) at which global symmetry breaking phenomena occur. Here and thereafter we use the notation of Sect. 3.1. Recall that $\mathcal{T}$ denotes the set of trivial solutions.

Definition 3.9 We say that a global symmetry breaking phenomenon occurs at the orbit $\mathcal{G}\left(\tilde{u}_{0}\right) \times\left\{\lambda_{0}\right\}$ if $\lambda_{0} \in G L O B$ and there exists $U \subset \mathbb{H} \times \mathbb{R}$ such that $\mathcal{G}\left(\tilde{u}_{0}\right) \times\left\{\lambda_{0}\right\} \subset U$ and $\mathcal{G}_{(u, \lambda)} \neq \mathcal{G}_{\left(\tilde{u}_{0}, \lambda_{0}\right)}$ for all $(u, \lambda) \in\left(U \cap\left(\nabla_{u} \Phi\right)^{-1}(0)\right) \backslash \mathcal{T}$. 
Note that since the group $\mathcal{G}$ acts trivially on the set of parameters $\lambda$, the condition $\mathcal{G}_{(u, \lambda)} \neq$ $\mathcal{G}_{\left(\tilde{u}_{0}, \lambda_{0}\right)}$ is equivalent to $\mathcal{G}_{u} \neq \mathcal{G}_{\tilde{u}_{0}}$. In particular we are interested in studying $\operatorname{SO}(N)$ symmetries of solutions. We say that the function $u$ satisfying $S O(N)_{u}=S O(N)$ is radially symmetric.

Our aim in this section is to prove the following characterisation of global symmetry breaking phenomena of solutions of (2.1):

Theorem 3.10 Consider the system (2.1) with the potential $F$ and $u_{0} \in \nabla F^{-1}(0)$ satisfying assumptions (B1)-(B4). Fix $\lambda_{0} \in \Lambda$ and suppose that $\sigma\left(\lambda_{0} A\right) \cap \sigma\left(-\Delta ; B^{N}\right) \backslash\{0\}=$ $\left\{\alpha_{j_{1}}, \ldots, \alpha_{j_{s}}\right\}$ and $\mathbb{V}_{-\Delta}\left(\alpha_{j_{i}}\right)^{S O(N)}=\{0\}$ for every $i=1, \ldots, s$. Then the global symmetry breaking phenomenon occurs at the orbit $\mathcal{G}\left(\tilde{u}_{0}\right) \times\left\{\lambda_{0}\right\}$.

Note that the assumption $\mathbb{V}_{-\Delta}\left(\alpha_{j_{i}}\right)^{S O(N)}=\{0\}$ means that there is no radially symmetric eigenfunction associated with $\alpha_{j_{i}}$.

To prove this theorem we first verify the following lemma:

Lemma 3.11 Fix $\lambda_{0} \in \Lambda$. Then there exists $U \subset \mathbb{H} \times \mathbb{R}$ such that $\mathcal{G}\left(\tilde{u}_{0}\right) \times\left\{\lambda_{0}\right\} \subset U$ and for all $(u, \lambda) \in\left(U \cap\left(\nabla_{u} \Phi\right)^{-1}(0)\right) \backslash \mathcal{T}$ there exists $\bar{u} \in \operatorname{ker} \nabla_{u}^{2} \Phi_{\mid \mathbb{H}_{1}^{\perp}}\left(\tilde{u}_{0}, \lambda_{0}\right) \backslash\{0\}$ such that $\mathcal{G}_{u} \subset \mathcal{G}_{\bar{u}}$.

Proof Consider $\mathbb{U}_{1}=\operatorname{im} \nabla_{u}^{2} \Phi_{\mid \mathbb{H}_{1}^{\perp}}\left(\tilde{u}_{0}, \lambda_{0}\right) \oplus \mathbb{H}_{1}$ and $\mathbb{U}_{2}=\operatorname{ker} \nabla_{u}^{2} \Phi_{\mid \mathbb{H}_{1}^{\perp}}\left(\tilde{u}_{0}, \lambda_{0}\right)$. Note that $\mathbb{H}=\mathbb{U}_{1} \oplus \mathbb{U}_{2}$ and the spaces $\mathbb{U}_{1}$ and $\mathbb{U}_{2}$ are $\mathcal{G}$-representations. For $u \in \mathbb{H}$ we put $u=$ $\left(u_{1}, u_{2}\right) \in \mathbb{U}_{1} \oplus \mathbb{U}_{2}$. In particular, since $\tilde{u}_{0} \in \mathbb{H}_{1}$, we identify this element with $\left(\tilde{u}_{0}, 0\right) \in$ $\mathbb{U}_{1} \oplus \mathbb{U}_{2}$.

The equation

$$
\nabla_{u} \Phi(u, \lambda)=0
$$

is equivalent to the system

$$
\begin{aligned}
& \pi_{1}\left(\nabla_{u} \Phi\left(u_{1}, u_{2}, \lambda\right)\right)=0, \\
& \pi_{2}\left(\nabla_{u} \Phi\left(u_{1}, u_{2}, \lambda\right)\right)=0,
\end{aligned}
$$

where $\pi_{1}: \mathbb{H} \rightarrow \mathbb{U}_{1}$ and $\pi_{2}: \mathbb{H} \rightarrow \mathbb{U}_{2}$ are $\mathcal{G}$-equivariant projections. Moreover, since $\mathcal{G}\left(\tilde{u}_{0}\right) \subset \mathbb{H}_{1} \subset \mathbb{U}_{1}$,

$$
\operatorname{dim} \operatorname{ker} \nabla_{u}^{2} \Phi_{\mid \mathbb{U}_{1}}\left(\tilde{u}_{0}, \lambda_{0}\right)=\operatorname{dim} \mathcal{G}\left(\tilde{u}_{0}\right),
$$

i.e. $\mathcal{G}\left(\tilde{u}_{0}\right)$ is a non-degenerate critical orbit of $\Phi\left(\cdot, \lambda_{0}\right) \mathbb{U}_{1}$. Therefore, by the equivariant implicit function theorem (see Theorem 5.1) applied to the functional $\Phi: \mathbb{U}_{1} \oplus\left(\mathbb{U}_{2} \times \mathbb{R}\right) \rightarrow \mathbb{R}$, the point $\left(0, \lambda_{0}\right)$ and the Eq. (3.8), there exist open sets $\mathcal{O}_{0} \subset \mathbb{U}_{2}, \mathcal{O}_{\lambda_{0}} \subset \mathbb{R}$ such that $0 \in \mathcal{O}_{0}, \lambda_{0} \in \mathcal{O}_{\lambda_{0}}$ and a $\mathcal{G}$-equivariant map $\tau: \mathcal{G}\left(\tilde{u}_{0}\right) \times \mathcal{O}_{0} \times \mathcal{O}_{\lambda_{0}} \rightarrow \mathbb{U}_{1}$ such that

(i) $\tau\left(u_{1}, 0, \lambda_{0}\right)=u_{1}$ for $u_{1} \in \mathcal{G}\left(\tilde{u}_{0}\right)$,

(ii) $\pi_{1}\left(\nabla_{u} \Phi\left(\tau\left(u_{1}, u_{2}, \lambda\right), u_{2}, \lambda\right)\right)=0$ if $u_{1} \in \mathcal{G}\left(\tilde{u}_{0}\right), u_{2} \in \mathcal{O}_{0}$ and $\lambda \in \mathcal{O}_{\lambda_{0}}$ and these are the only solutions of $\pi_{1}\left(\nabla_{u} \Phi\left(u_{1}, u_{2}, \lambda\right)\right)=0$ near the orbit if $u_{2} \in \mathcal{O}_{0}$ and $\lambda \in \mathcal{O}_{\lambda_{0}}$.

Hence all the solutions of the Eq. (3.8), and consequently the solutions of (3.9) and (3.7), can have (in the neighbourhood of the orbit) only the following isotropy groups:

$$
\mathcal{G}_{\left(\tau\left(u_{1}, u_{2}, \lambda\right), u_{2}, \lambda\right)}=\mathcal{G}_{\tau\left(u_{1}, u_{2}, \lambda\right)} \cap \mathcal{G}_{u_{2}} \cap \mathcal{G}_{\lambda}=\mathcal{G}_{\tau\left(u_{1}, u_{2}, \lambda\right)} \cap \mathcal{G}_{u_{2}} \subset \mathcal{G}_{u_{2}} .
$$


Denote this neighbourhood by $U$. More precisely, $U=\operatorname{int}\left(\tau\left(\mathcal{G}\left(\tilde{u}_{0}\right) \times \mathcal{O}_{0} \times \mathcal{O}_{\lambda_{0}}\right)\right) \times$ $\mathcal{O}_{0} \times \mathcal{O}_{\lambda_{0}}$. To finish the proof observe that in the case $u_{2}=0$ we have $\left(\tau\left(u_{1}, 0, \lambda\right), 0, \lambda\right) \in$ $\mathbb{U}_{1} \times\{0\} \times \mathbb{R}$ for $u_{1} \in \mathcal{G}\left(\tilde{u}_{0}\right), \lambda \in \mathcal{O}_{\lambda_{0}}$. Considering only the solutions of (3.8) and observing that such solutions in $\mathbb{U}_{1} \times\{0\} \times \mathbb{R}$ are the trivial ones, we obtain $\left(\tau\left(u_{1}, 0, \lambda\right), 0, \lambda\right) \in \mathcal{T}$, which completes the proof.

Lemma 3.11 generalises a lemma due to Dancer from [4]. Dancer's result states that if the kernel of the second derivative of the functional at a bifurcation point does not contain nonzero radially-symmetric elements, then at a neighbourhood of this point all nontrivial solutions are not radial. This lemma cannot be applied to prove Theorem 3.10 in the case $\operatorname{dim} \mathcal{G}\left(\tilde{u}_{0}\right)>0$, since $\operatorname{ker} \nabla_{u}^{2} \Phi\left(\tilde{u}_{0}, \lambda_{0}\right)$ contains constant (and therefore radially symmetric) functions from the space tangent to the orbit.

Proof of Theorem 3.10 Note that by the assumptions of the theorem, from Remark 5.11 it follows that the assumption (C1) is satisfied. Therefore Theorem 3.5 implies that $\lambda_{0} \in$ $G L O B$. Moreover, from Corollary 2.3 we have

$$
\begin{aligned}
\operatorname{ker} \nabla_{u}^{2} \Phi_{\mid \mathbb{H}_{1}^{\perp}}\left(\tilde{u}_{0}, \lambda_{0}\right) & =\operatorname{ker}\left(I d-L_{\lambda_{0} A}\right) \cap \mathbb{H}_{1}^{\perp} \\
& =\mathbb{V}_{-\Delta}\left(\alpha_{j_{1}}\right)^{\mu_{\lambda_{0} A}\left(\alpha_{j_{1}}\right)} \oplus \cdots \oplus \mathbb{V}_{-\Delta}\left(\alpha_{j_{s}}\right)^{\mu_{\lambda_{0} A}\left(\alpha_{j_{s}}\right)} .
\end{aligned}
$$

Since $\alpha_{j_{1}}, \ldots, \alpha_{j_{s}} \neq 0$ are such that $\mathbb{V}_{-\Delta}\left(\alpha_{j_{i}}\right)^{S O(N)}=\{0\}$ for every $i=1, \ldots, s$, we conclude that

$$
\operatorname{ker} \nabla_{u}^{2} \Phi_{\mid \mathbb{H}_{1}^{\perp}}\left(\tilde{u}_{0}, \lambda_{0}\right)^{S O(N)}=\{0\} .
$$

Lemma 3.11 yields that there exists $U \subset \mathbb{H} \times \mathbb{R}$ such that if $\nabla_{u} \Phi(u, \lambda)=0$ and $(u, \lambda) \in U \backslash \mathcal{T}$ then there exists $\bar{u} \in \operatorname{ker} \nabla_{u}^{2} \Phi_{\mid \mathbb{H}_{1}^{\perp}}\left(\tilde{u}_{0}, \lambda_{0}\right) \backslash\{0\}$ such that $\mathcal{G}_{u} \subset \mathcal{G}_{\bar{u}}$. Since $\mathcal{G}_{\tilde{u}_{0}}=\{e\} \times S O(N)$, to prove that $\mathcal{G}_{u} \neq \mathcal{G}_{\tilde{u}_{0}}$ it suffices to note that the isotropy group of $\bar{u}$ is not of the form $H \times S O(N)$, where $H \in \overline{\operatorname{sub}}(\Gamma)$. Indeed, if $\mathcal{G}_{\bar{u}}=H \times S O(N)$, then $\bar{u}\left(\alpha^{-1} x\right)=\bar{u}(x)$ for every $\alpha \in S O(N), x \in B^{N}$, i.e. $S O(N)_{\bar{u}}=S O(N)$ and therefore from (3.10) we obtain $\bar{u}=0$, which contradicts $\bar{u} \in \operatorname{ker} \nabla_{u}^{2} \Phi_{\mid \mathbb{H}_{1}^{\perp}}\left(\tilde{u}_{0}, \lambda_{0}\right) \backslash\{0\}$.

Note that if the assumptions of Theorem 3.10 are satisfied, i.e. $\operatorname{ker} \nabla_{u}^{2} \Phi_{\mid \mathbb{H}_{1}^{\perp}}\left(\tilde{u}_{0}, \lambda_{0}\right)^{S O(N)}=$ $\{0\}$, then there is a neighbourhood $U$ of the bifurcation orbit such that all nontrivial solutions from $U$ are non-radial. In other words, in Theorem 3.10 we obtain a connected family of orbits of non-radial solutions bifurcating from the set of radial ones.

Remark 3.12 Let $\lambda_{0} \in B I F$. By the proof of Lemma 3.11 we deduce that there is a neighbourhood of the orbit $\mathcal{G}\left(\tilde{u}_{0}\right) \times\left\{\lambda_{0}\right\}$ such that all nontrivial solutions of $\nabla_{u} \Phi(u, \lambda)=0$ can have only isotropy groups of the form $\mathcal{G}_{\tau\left(u_{1}, u_{2}, \lambda\right)} \cap \mathcal{G}_{u_{2}}$. Note that $u_{1} \in \mathcal{G}\left(\tilde{u}_{0}\right)$ and hence $\mathcal{G}_{u_{1}}=\{e\} \times S O(N)$.

Consider the additional assumption:

$$
\operatorname{ker} \nabla_{u}^{2} \Phi_{\mid \mathbb{H}_{1}^{\perp}}\left(\tilde{u}_{0}, \lambda_{0}\right)^{S O(N)}=\operatorname{ker} \nabla_{u}^{2} \Phi_{\mid \mathbb{H}_{1}^{\perp}}\left(\tilde{u}_{0}, \lambda_{0}\right) .
$$

Then $\mathcal{G}_{u_{2}}=\Gamma_{u_{2}} \times S O(N)$. Therefore by the proof of Lemma 3.11, and since a $\mathcal{G}$-equivariant function $\tau$ increases isotropy groups (i.e. $\left.\mathcal{G}_{\left(u_{1}, u_{2}, \lambda\right)} \subset \mathcal{G}_{\tau\left(u_{1}, u_{2}, \lambda\right)}\right)$, we have

$$
\begin{aligned}
\mathcal{G}_{u_{1}} \cap \mathcal{G}_{u_{2}} & =(\{e\} \times S O(N)) \cap\left(\Gamma_{u_{2}} \times S O(N)\right) \\
& =\{e\} \times S O(N) \subset \mathcal{G}_{\tau\left(u_{1}, u_{2}, \lambda\right)} \cap \mathcal{G}_{u_{2}},
\end{aligned}
$$


i.e. solutions of $\nabla_{u} \Phi(u, \lambda)=0$ in the neighbourhood of the orbit $\mathcal{G}\left(\tilde{u}_{0}\right) \times\left\{\lambda_{0}\right\}$ have isotropy groups of the form $H \times S O(N)$, where $H \in \overline{\operatorname{sub}}(\Gamma)$. Hence all solutions from the neighbourhood of the orbit are radial.

Remark 3.13 Fix $\lambda_{0} \in \Lambda$ and suppose that $\sigma\left(\lambda_{0} A\right) \cap \sigma\left(-\Delta ; B^{N}\right) \backslash\{0\}=\left\{\alpha_{j_{1}}, \ldots, \alpha_{j_{s}}\right\}$ are such that $\alpha_{j_{1}}, \ldots, \alpha_{j_{s}} \notin \mathcal{A}_{0}$, where $\mathcal{A}_{0}$ is defined in Sect. 5.4. Then from Remark 5.12 it follows that $\mathbb{V}_{-\Delta}\left(\alpha_{j_{i}}\right)^{S O(N)}=\{0\}$ and therefore the assumptions of Theorem 3.10 are satisfied. Hence the global symmetry breaking phenomenon occurs at the orbit $\mathcal{G}\left(\tilde{u}_{0}\right) \times\left\{\lambda_{0}\right\}$.

\section{Examples}

In this section we discuss a few examples in order to illustrate the abstract results proved in the previous section. Using the properties of the eigenspaces of the Laplace operator (with Neumann boundary conditions) on the ball, we verify assumptions (C1)-(C3). More precisely we apply the material collected in Sect. 5.4.

Example 1 Consider the system (2.1) for $N=2$ with a potential $F$ and $u_{0} \in \nabla F^{-1}(0)$ satisfying assumptions (B1)-(B4). Assume that $\lambda_{0} \in \mathbb{R} \backslash\{0\}$ and $\sigma\left(\lambda_{0} A\right) \cap \sigma\left(-\Delta ; B^{2}\right) \backslash\{0\}=$ $\{\alpha\}$, where $\sqrt{\alpha}$ is not a root of $J_{0}^{\prime}(x)=0$ for $J_{0}$ being the Bessel function of order 0 . Following the notation of Sect. 5.4 it means that $\alpha \notin \mathcal{A}_{0}$.

In this situation, from Theorem 5.9 and Fact 5.10 the assumption $(\mathrm{C} 1)$ of Sect. 3 is satisfied. By Theorem 3.5 we obtain that a global bifurcation occurs from the orbit $\mathcal{G}\left(\tilde{u}_{0}\right) \times\left\{\lambda_{0}\right\}$.

Moreover, from Remark 5.12 it follows that $\mathbb{V}_{-\Delta}(\alpha)^{S O(2)}=\{0\}$. Then by Theorem 3.10 the global symmetry breaking occurs at the orbit $\mathcal{G}\left(\tilde{u}_{0}\right) \times\left\{\lambda_{0}\right\}$.

Example 2 Consider the system (2.1) for $N=2$ with the potential $F$ and $u_{0} \in \nabla F^{-1}(0)$ satisfying assumptions (B1)-(B4). Assume that $\lambda_{0} \in \mathbb{R} \backslash\{0\}, \sigma\left(\lambda_{0} A\right) \cap \sigma\left(-\Delta ; B^{2}\right) \backslash\{0\}=$ $\left\{\alpha_{1}, \ldots, \alpha_{s}\right\}$ and there exists $i \in\{1, \ldots, s\}$ such that $\sqrt{\alpha_{i}}$ is not a root of $J_{0}^{\prime}(x)=0$.

As in Example 1, a global bifurcation occurs from the orbit $\mathcal{G}\left(\tilde{u}_{0}\right) \times\left\{\lambda_{0}\right\}$. If moreover $\alpha_{i} \notin \mathcal{A}_{0}$ for all $i \in\{1, \ldots, s\}$, then the global symmetry breaking occurs at the orbit $\mathcal{G}\left(\tilde{u}_{0}\right) \times\left\{\lambda_{0}\right\}$.

Example 3 Consider the system (2.1) for $N=3$ with the potential $F$ and $u_{0} \in \nabla F^{-1}(0)$ satisfying assumptions (B1)-(B4). Assume that $\lambda_{0} \in \mathbb{R} \backslash\{0\}, \sigma\left(\lambda_{0} A\right) \cap \sigma\left(-\Delta ; B^{3}\right) \backslash\{0\}=$ $\left\{\alpha_{1}, \ldots, \alpha_{s}\right\}$ and there exists $i \in\{1, \ldots, s\}$ such that $\sqrt{\alpha_{i}}$ is not a solution of the equation:

$$
J_{\frac{1}{2}}^{\prime}(x)-\frac{1}{2 x} J_{\frac{1}{2}}(x)=0,
$$

where $J_{\frac{1}{2}}$ is the Bessel function of order $\frac{1}{2}$. Therefore $\alpha_{i} \notin \mathcal{A}_{0}$.

In this situation, since $\mathcal{H}_{l}^{3} \subset \mathbb{V}_{-\Delta}\left(\alpha_{i}\right)$ for some $l>0$ (by Fact 5.10), the assumption (C1) is satisfied and from Theorem 3.5 we obtain that a global bifurcation occurs from the orbit $\mathcal{G}\left(\tilde{u}_{0}\right) \times\left\{\lambda_{0}\right\}$.

Moreover, if $\alpha_{i} \notin \mathcal{A}_{0}$ for all $i \in\{1, \ldots, s\}$, then from Remark 5.12 we conclude that $\mathbb{V}_{-\Delta}\left(\alpha_{i}\right)^{S O(3)}=\{0\}$ for all $i \in\{1, \ldots, s\}$. Therefore, by Theorem 3.10 it follows that the global symmetry breaking occurs at the orbit $\mathcal{G}\left(\tilde{u}_{0}\right) \times\left\{\lambda_{0}\right\}$. 
Example 4 Consider the system (2.1) with the potential $F$ and $u_{0} \in \nabla F^{-1}(0)$ satisfying assumptions (B1)-(B4). Assume that $\lambda_{0} \in \mathbb{R} \backslash\{0\}$ and that $\sigma\left(\lambda_{0} A\right) \cap \sigma\left(-\Delta ; B^{N}\right) \backslash\{0\}=$ $\left\{\alpha_{1}, \ldots, \alpha_{s}\right\}$, where $\sqrt{\alpha_{i}}$ is a solution of the equation

$$
J_{\frac{N-2}{2}}^{\prime}(x)-\frac{N-2}{2 x} J_{\frac{N-2}{2}}(x)=0
$$

for every $i \in\{1, \ldots, s\}$.

If there exists $i \in\{1, \ldots, s\}$ such that $\operatorname{dim} \mathbb{V}_{-\Delta}\left(\alpha_{i}\right)>1$ then the assumption (C1) is satisfied and by Theorem 3.5 we obtain that a global bifurcation occurs from the orbit $\mathcal{G}\left(\tilde{u}_{0}\right) \times\left\{\lambda_{0}\right\}$.

If $\operatorname{dim} \mathbb{V}_{-\Delta}\left(\alpha_{i}\right)=1$ for all $i \in\{1, \ldots, s\}$, then we assume additionally that $\sum_{i=1}^{s} \mu_{\lambda_{0} A}\left(\alpha_{i}\right)-\mu_{A}(0)$ is an odd number. In this situation the assumption $(\mathrm{C} 2)$ is satisfied and by Theorem 3.5 we obtain that a global bifurcation occurs from the orbit $\mathcal{G}\left(\tilde{u}_{0}\right) \times\left\{\lambda_{0}\right\}$.

Note that, if $\operatorname{dim} \mathbb{V}_{-\Delta}\left(\alpha_{i}\right)=1$ for all $i \in\{1, \ldots, s\}$, then $\operatorname{ker}\left(I d-L_{\lambda_{0} A}\right)^{S O(N)}=$ $\operatorname{ker}\left(I d-L_{\lambda_{0} A}\right)$ (see Remark 5.11(2)). Therefore, from Remark 3.12, we conclude that all nontrivial solutions at a neighbourhood of $\mathcal{G}\left(\tilde{u}_{0}\right) \times\left\{\lambda_{0}\right\}$ (bifurcating from this orbit) are radial, i.e. there is no symmetry breaking at the orbit.

Example 5 Consider the system (2.1) with the potential $F$ and $u_{0} \in \nabla F^{-1}(0)$ satisfying assumptions (B1)-(B4). Assume that $\lambda_{0}=0$.

If $m-\operatorname{dim} \operatorname{ker} A$ is odd, then the assumption (C3) is satisfied and we obtain a global bifurcation from the orbit $\mathcal{G}\left(\tilde{u}_{0}\right) \times\{0\}$. If $m-\operatorname{dim} \operatorname{ker} A>0$, then Theorem 3.8 implies a local bifurcation from the orbit $\mathcal{G}\left(\tilde{u}_{0}\right) \times\{0\}$.

As in Example 4, it is easy to see that all nontrivial solutions at a neighbourhood of the orbit are radial.

Acknowledgements The authors wish to express their gratitude to the referee for many propositions which allowed to improve the paper.

Open Access This article is distributed under the terms of the Creative Commons Attribution 4.0 International License (http://creativecommons.org/licenses/by/4.0/), which permits unrestricted use, distribution, and reproduction in any medium, provided you give appropriate credit to the original author(s) and the source, provide a link to the Creative Commons license, and indicate if changes were made.

\section{Appendix}

In the following section, to make the paper self-contained, we collect some classical definitions and facts which we use to prove our main results.

\subsection{The equivariant implicit function theorem}

Below we reformulate an equivariant version of the implicit function theorem in infinite dimensional spaces, due to Dancer (see [5], paragraph 3).

Theorem 5.1 Let $G$ be a compact Lie group and suppose that

(i) $\mathbb{H}_{1}, \mathbb{H}_{2}$ are Hilbert spaces, which are orthogonal $G$-representations,

(ii) $\Phi: \mathbb{H}_{1} \oplus \mathbb{H}_{2} \rightarrow \mathbb{R}$ is a $G$-invariant functional of class $C^{2}$,

(iii) there is $v_{0} \in \mathbb{H}_{2}$ such that $\nabla_{u}^{2} \Phi\left(u, v_{0}\right)$ is Fredholm for every $u \in \mathbb{H}_{1}$, there is $u_{0} \in \mathbb{H}_{1}$ such that $\nabla_{u} \Phi\left(u_{0}, v_{0}\right)=0$ and $G\left(u_{0}\right)$ is a non-degenerate critical orbit of $\Phi\left(\cdot, v_{0}\right)$. 
Then there exist $\delta>0$ and a continuous $G$-invariant map $\tau: G\left(u_{0}\right) \times B_{\delta}\left(v_{0}, \mathbb{H}_{2}\right) \rightarrow \mathbb{H}_{1}$ such that

(1) $\tau\left(u, v_{0}\right)=$ u on $G\left(u_{0}\right)$,

(2) $\nabla_{u} \Phi(\tau(u, v), v)=0$ if $u \in G\left(u_{0}\right)$ and $v \in B_{\delta}\left(v_{0}, \mathbb{H}_{2}\right)$ and these are the only solutions of $\nabla_{u} \Phi(u, v)=0$ near $G\left(u_{0}\right)$ if $v \in B_{\delta}\left(v_{0}, \mathbb{H}_{2}\right)$,

(3) for each $v \in B_{\delta}\left(v_{0}, \mathbb{H}_{2}\right)$, the map $u \mapsto \tau(u, v)$ is one-to-one.

\subsection{Equivariant Conley index}

In this subsection we collect properties of the equivariant Conley index. For a more detailed exposition we refer to [2,8] in the finite dimensional case and to [13] for the infinite dimensional case. Note that the Conley index (in the finite dimensional as well as in the infinite dimensional case) is defined for an arbitrary compact Lie group $G$. In our paper we use it in special cases $G=\mathcal{G}=\Gamma \times S O(N)$ and $G=S O(N)$.

Let $G$ be a compact Lie group and suppose that $\Omega$ is a $G$-invariant subset of a finite dimensional $G$-representation $\mathbb{V}$. The $G$-equivariant Conley index of an isolating neighbourhood of a (local) flow is defined as the $G$-homotopy type of a pointed $G$-space, see [2,8]. If $f: \Omega \rightarrow \mathbb{V}$ is a $G$-equivariant map of class $C^{1}$, then it generates a local $G$-flow $\eta$, such that $\eta\left(x_{0}, \cdot\right)$ is the local solution of the problem $y^{\prime}(t)=f(y(t)), y(0)=x_{0}$. Moreover, if $S$ is an isolated invariant set of the flow, then there exists an isolating neighbourhood for this set. To simplify the notation in the main part of our paper, we denote by $C I_{G}(S, f)$ the Conley index of an isolating neighbourhood of the isolated invariant set $S$ of the flow generated by $f$.

Put $S^{\mathbb{V}}=D_{1}(0, \mathbb{V}) / \partial D_{1}(0, \mathbb{V})$ and denote by $\left[S^{\mathbb{V}}\right]_{G}$ the $G$-homotopy type of a pointed $G$-space $S^{\mathbb{V}}$. From the definition of the Conley index and the Hartman-Grobman theorem it follows (see also [26]):

Theorem 5.2 Let $f: \mathbb{V} \rightarrow \mathbb{R}$ be a $G$-invariant map of class $C^{2}$ and suppose that $v_{0} \in \mathbb{V}$ is such that $G\left(v_{0}\right)=\left\{v_{0}\right\}, \nabla f\left(v_{0}\right)=0$ and $\operatorname{det} \nabla^{2} f\left(v_{0}\right) \neq 0$. Then $C I_{G}\left(\left\{v_{0}\right\},-\nabla f\right)=$ $\left[S^{\mathbb{V}^{-}}\right]_{G}$, where $\mathbb{V}^{-}$is the direct sum of eigenspaces of $\nabla^{2} f\left(v_{0}\right)$ corresponding to the negative eigenvalues.

The following theorem is a direct consequence of the Continuation Property of the Conley index, see [2]:

Theorem 5.3 (Homotopy invariance) Let $v_{0} \in \mathbb{V}$ be such that $G\left(v_{0}\right)=\left\{v_{0}\right\}$ and suppose that $f \in C^{2}(\mathbb{V} \times[0,1], \mathbb{R})$ is $G$-invariant. If $\nabla_{v} f\left(v_{0}, t\right)=0$ and $\operatorname{det} \nabla_{v}^{2} f\left(v_{0}, t\right) \neq 0$ for every $t \in[0,1]$, then

$$
C I_{G}\left(\left\{v_{0}\right\}, \nabla_{v} f(\cdot, 0)\right)=C I_{G}\left(\left\{v_{0}\right\}, \nabla_{v} f(\cdot, 1)\right) .
$$

The Conley index of a flow generated by a gradient map is the homotopy type of a pointed finite $G$-CW-complex, see Proposition 5.6 of [8] for the proof. With a $G$-homotopy type of a pointed finite $G$-CW-complex $X$ one can associate a $G$-equivariant Euler characteristic $\chi_{G}(X)$, which is an element of the Euler $\operatorname{ring} U(G)$ with the unit $\mathbb{I}=\chi_{G}\left(G / G^{+}\right)$. The actions in $U(G)$ are defined by

$$
\begin{aligned}
\chi_{G}(X)+\chi_{G}(Y) & =\chi_{G}(X \vee Y), \\
\chi_{G}(X) \star \chi_{G}(Y) & =\chi_{G}(X \wedge Y),
\end{aligned}
$$

where $X \vee Y$ is the wedge sum and $X \wedge Y$ is the smash product of pointed finite $G$-CW-complexes $X, Y$. It is known that $(U(G),+)$ is a free abelian group with basis 
$\chi_{G}\left(G / H^{+}\right),(H)_{G} \in \overline{\operatorname{sub}}[G]$ (by $\overline{\operatorname{sub}}[G]$ we denote the set of conjugacy classes $(H)_{G}$ of closed subgroups of the group $G$ ). A more detailed exposition of this theory can be found for example in [28,29].

The following theorem is an immediate consequence of Lemma 3.4 of [6]:

Theorem 5.4 If the group $G$ is connected and $\mathbb{V}$ is a nontrivial $G$-representation, then $\chi_{G}\left(S^{\mathbb{V}}\right) \neq \mathbb{I} \in U(G)$.

Consider the potential $\varphi: \mathbb{V} \times \mathbb{R} \rightarrow \mathbb{R}$ and assume that for $\lambda_{-}, \lambda_{+} \in \mathbb{R}$ the critical orbit $G\left(\tilde{u}_{0}\right)$ of $\varphi\left(\cdot, \lambda_{ \pm}\right)$is non-degenerate. In Sect. 3 we compare equivariant Conley indices $C I_{G}\left(G\left(\tilde{u}_{0}\right),-\nabla \varphi\left(\cdot, \lambda_{ \pm}\right)\right)$. Following the method introduced in [18], we want to reduce this problem to comparing the Euler characteristics of the Conley indices of potentials restricted to the space orthogonal to the orbit. This method bases on the relation between Conley indices obtained with the use of the smash product over $G_{\tilde{u}_{0}}$. To make our paper self-contained, we recall the relevant material.

Let $H \in \overline{\operatorname{sub}}(G)$ and $\mathbb{X}$ be a pointed $H$-space with a base point $*$. Denote by $G^{+}$the group $G$ with disjoint base point added. The smash product of $G^{+}$and $\mathbb{X}$ is defined by $G^{+} \wedge \mathbb{X}=\left(G^{+} \times \mathbb{X}\right) /\left(G^{+} \vee \mathbb{X}\right)=G \times \mathbb{X} / G \times\{*\}$. The space $G^{+} \wedge \mathbb{X}$ is an $H$-space with an action given by $(h,[g, x]) \mapsto\left[g h^{-1}, h x\right]$. The orbit space of this action is called the smash product over $H$ and denoted by $G^{+} \wedge_{H} \mathbb{X}$, see [29]. Note that formula $\left(g^{\prime},[g, x]\right) \mapsto\left[g^{\prime} g, x\right]$ induces a $G$-action on $G^{+} \wedge_{H} \mathbb{X}$ and therefore this is a pointed $G$-space.

Theorem 5.5 (Theorem 3.1 of [18]) Let $\Omega$ be an open, G-invariant subset of $\mathbb{V}$. Let $\varphi \in C^{2}(\Omega, \mathbb{R})$ be a $G$-invariant map and $\tilde{u}_{0} \in \Omega$. Suppose that the orbit $G\left(\tilde{u}_{0}\right) \subset$ $(\nabla \varphi)^{-1}(0)$ is non-degenerate. Define $\phi=\varphi_{\mid T_{\tilde{u}_{0}}^{\perp} G\left(\tilde{u}_{0}\right)}$. Then $C I_{G}\left(G\left(\tilde{u}_{0}\right),-\nabla \varphi\right)=G^{+} \wedge_{H}$ $C I_{H}\left(\left\{\tilde{u}_{0}\right\},-\nabla \phi\right)$, where $H=G_{\tilde{u}_{0}}$.

It is known that if the pair $(G, H)$ is admissible, then coordinates of the Euler characteristics of the $H$-CW-complex $X$ and the $G$-CW-complex $G \wedge_{H} X$ coincide. More precisely, Theorem 2.3 of [18] states that if $\chi_{H}(X)=\sum_{(K)_{H} \in \overline{\operatorname{sub}}[H]} n_{K} \cdot \chi_{H}\left(H / K^{+}\right)$, then $\chi_{G}\left(G^{+} \wedge_{H} X\right)=\sum_{(K)_{H} \in \overline{\operatorname{sub}}[H]} n_{K} \cdot \chi_{G}\left(G / K^{+}\right)$. From this relation and Theorem 5.5, we obtain the following fact:

Fact 5.6 Let $\Omega \subset \mathbb{V}$ be an open and $G$-invariant subset and $\varphi \in C^{2}(\Omega \times \mathbb{R}, \mathbb{R})$ be $G$ invariant. Moreover, let $\lambda_{-}, \lambda_{+} \in \mathbb{R}$ and $G\left(\tilde{u}_{0}\right) \subset\left(\nabla_{u} \varphi\left(\cdot, \lambda_{ \pm}\right)\right)^{-1}(0)$ be a non-degenerate critical orbit. Set $\phi=\varphi_{\mid T_{\tilde{u}_{0}}^{\perp} G\left(\tilde{u}_{0}\right)}$. If the pair $\left(G, G_{\tilde{u}_{0}}\right)$ is admissible and

$$
\chi_{G_{\tilde{u}_{0}}}\left(C I_{G_{\tilde{u}_{0}}}\left(\left\{\tilde{u}_{0}\right\},-\nabla_{u} \phi\left(\cdot, \lambda_{-}\right)\right) \neq \chi_{\tilde{u}_{0}}\left(C I_{G_{\tilde{u}_{0}}}\left(\left\{\tilde{u}_{0}\right\},-\nabla_{u} \phi\left(\cdot, \lambda_{+}\right)\right)\right.\right.
$$

then

$$
\chi_{G}\left(C I_{G}\left(G\left(\tilde{u}_{0}\right),-\nabla_{u} \varphi\left(\cdot, \lambda_{-}\right)\right)\right) \neq \chi_{G}\left(C I_{G}\left(G\left(\tilde{u}_{0}\right),-\nabla_{u} \varphi\left(\cdot, \lambda_{+}\right)\right)\right) .
$$

In the following we briefly describe the infinite dimensional version of the $G$-equivariant Conley index of an isolated invariant set of a (local) $G-\mathcal{L} \mathcal{S}$-flow. This index is defined as a $G$-homotopy type of a $G$-equivariant spectrum, see [13]. Below we recall this definition in a special case. Namely, since in our computation we use the $G$-equivariant gradient maps of the form of a completely continuous perturbation of the identity, we consider only flows generated by such maps.

We start with a definition of a $G$-spectrum. Fix a sequence $\xi=\left(\mathbb{V}_{n}\right)_{n=0}^{\infty}$ of finitedimensional orthogonal $G$-representations. A pair of sequences:

$$
\mathcal{E}=\left(\left(\mathcal{E}_{n}\right)_{n=n(\mathcal{E})}^{\infty},\left(\epsilon_{n}\right)_{n=n(\mathcal{E})}^{\infty}\right)
$$


of finite pointed $G$-CW-complexes $\mathcal{E}_{n}$ and morphisms $\epsilon_{n}: S^{\mathbb{V}_{n}} \wedge \mathcal{E}_{n} \rightarrow \mathcal{E}_{n+1}$ is called a $G$-spectrum if there exists $n_{0}>n(\mathcal{E})$ such that $\epsilon_{n}$ is a $G$-homotopy equivalence for all $n \geq n_{0}$.

Let $\mathbb{H}$ be an infinite-dimensional, separable Hilbert space, which is an orthogonal $G$ representation. Assume that $\mathbb{H}=\operatorname{cl}\left(\bigoplus_{n=0}^{\infty} \mathbb{H}_{n}\right)$, where all subspaces $\mathbb{H}_{n}$ are disjoint finitedimensional $G$-representations and put $\mathbb{H}^{n}=\bigoplus_{k=0}^{n} \mathbb{H}_{k}$. Let $\xi \in C^{1}(\mathbb{H}, \mathbb{R})$ be a $G$-invariant functional such that $\nabla \xi: \mathbb{H} \rightarrow \mathbb{H}$ is a completely continuous, $G$-equivariant operator. Put $f=I d-\nabla \xi$.

Denote by $\tau_{n}: \mathbb{H} \rightarrow \mathbb{H}^{n}$ the $G$-equivariant orthogonal projection. Define $f_{n}: \mathbb{H}^{n} \rightarrow \mathbb{H}^{n}$ by setting $f_{n}(u)=u-\tau_{n}(\nabla \xi(u))$ for any $u \in \mathbb{H}^{n}$. It is known (see [13]) that if $X$ is an isolating neighbourhood for the flow generated by $f$, then $X_{n}=X \cap \mathbb{H}^{n}$ is an isolating neighbourhood for the flow generated by $f_{n}$, for $n$ sufficiently large. It follows that $X_{n}$ admits an index pair $\left(Y_{n}, Z_{n}\right)$ with respect to the flow generated by $f_{n}$, and consequently, $C I_{G}\left(X_{n}, f_{n}\right)=\left[Y_{n} / Z_{n}\right]_{G}$. From the continuation property of the Conley index, the sequence $\left(\mathcal{E}_{n}\right)_{n=n_{0}}^{\infty}=\left(Y_{n} / Z_{n}\right)_{n=n_{0}}^{\infty}$ uniquely determines the $G$-homotopy type of a spectrum. The $G$ equivariant Conley index of $X$ with respect to the flow generated by $f$, denoted by $\mathcal{C I}_{G}(X, f)$, is defined as this $G$-homotopy type.

As in the finite dimensional case, we use the simplified notation $\mathcal{C I}_{G}(S, f)$ for the Conley index of an isolating neighbourhood of the isolated invariant set $S$ of the flow generated by $f$.

\subsection{The degree for equivariant gradient maps}

In this subsection we recall the definition and basic properties of the degree for $G$-equivariant gradient maps of the form of a completely continuous perturbation of the identity, denoted by $\nabla_{G}-\operatorname{deg}(\cdot, \cdot)$, defined by Rybicki [22]. This degree is an element of the Euler ring $U(G)$. Let $\mathbb{H}$ be an infinite-dimensional, separable Hilbert space which is an orthogonal $G$-representation. We keep the notation of the previous section, namely we consider finite dimensional subrepresentations $\mathbb{H}^{n}$ of $\mathbb{H}$ and the projections $\tau_{n}: \mathbb{H} \rightarrow \mathbb{H}^{n}$.

Assume that $\Omega \subset \mathbb{H}$ is an open, bounded and $G$-invariant subset and $\xi \in C^{1}(\mathbb{H}, \mathbb{R})$ is a $G$-invariant map such that

(1) $\nabla \xi: \mathbb{H} \rightarrow \mathbb{H}$ is a completely continuous, $G$-equivariant operator,

(2) $0 \notin(I d-\nabla \xi)^{-1}(\partial \Omega)$.

The degree of Rybicki is defined with the use of the degree for finite dimensional equivariant gradient maps introduced by Gęba [8], also being an element of the Euler ring $U(G)$. For simplicity of notation, we use the same symbol $\nabla_{G^{-}} \operatorname{deg}(\cdot, \cdot)$ for both degrees.

Restricting $I d-\nabla \xi$ to $\mathbb{H}^{n}$, one can study Gęba's degree of these restrictions. It appears (see [22]) that it stabilises for $n$ sufficiently large, i.e. there is $n_{0}$ such that

$$
\nabla_{G^{-}} \operatorname{deg}\left(\tau_{n}(I d-\nabla \xi), \Omega \cap \mathbb{H}^{n}\right)=\nabla_{G^{-}} \operatorname{deg}\left(\tau_{n_{0}}(I d-\nabla \xi), \Omega \cap \mathbb{H}^{n_{0}}\right)
$$

for every $n \geq n_{0}$.

Using this equality one can define the degree for infinite dimensional equivariant gradient maps. More precisely, fix $n_{0}$ as in the above lemma and define the degree for $G$-equivariant gradient maps of $I d-\nabla \xi$ on $\Omega$ by

$$
\nabla_{G^{-}} \operatorname{deg}(I d-\nabla \xi, \Omega)=\nabla_{G^{-}} \operatorname{deg}\left(\tau_{n_{0}}(I d-\nabla \xi), \Omega \cap \mathbb{H}^{n_{0}}\right) .
$$

A possible application of this degree is studying phenomena of global bifurcations and, in particular, an equivariant version of the Rabinowitz alternative. Let us make this more 
precise. Consider a family of $G$-invariant functionals $\Phi \in C^{2}(\mathbb{H} \times \mathbb{R}, \mathbb{R})$ and suppose that there is $u_{0} \in \mathbb{H}$ such that $\nabla_{u} \Phi\left(u_{0}, \lambda\right)=0$ for every $\lambda \in \mathbb{H}$. The invariance of $\Phi$ implies that $G\left(u_{0}\right) \subset\left(\nabla_{u} \Phi(\cdot, \lambda)\right)^{-1}(0)$ for every $\lambda \in \mathbb{H}$. We call the elements of $G\left(u_{0}\right) \times \mathbb{R}$ the trivial solutions of $\nabla_{u} \Phi(u, \lambda)=0$.

Assume additionally that $\nabla_{u} \Phi(u, \lambda)=u-\nabla_{u} \zeta(u, \lambda)$, where $\nabla_{u} \zeta: \mathbb{H} \times \mathbb{R} \rightarrow \mathbb{H}$ is a completely continuous, $G$-equivariant operator. Consider $\lambda_{0} \in \mathbb{R}$ such that the orbit $G\left(u_{0}\right)$ is degenerate in $\left(\nabla_{u} \Phi\left(\cdot, \lambda_{0}\right)\right)^{-1}(0)$. Suppose that there is $\varepsilon>0$ such that $G\left(u_{0}\right)$ is nondegenerate in $\left(\nabla_{u} \Phi(\cdot, \lambda)\right)^{-1}(0)$ for every $\lambda \in\left[\lambda_{0}-\varepsilon, \lambda_{0}+\varepsilon\right] \backslash\left\{\lambda_{0}\right\}$. Fix a $G$-invariant open set $\Omega \subset \mathbb{H}$ such that $\left(\nabla_{u} \Phi\left(\cdot, \lambda_{0} \pm \varepsilon\right)\right)^{-1}(0) \cap \Omega=G\left(u_{0}\right)$. Below we formulate an equivariant version of the Rabinowitz alternative.

Theorem 5.7 If $\nabla_{G^{-}} \operatorname{deg}\left(\nabla_{u} \Phi\left(\cdot, \lambda_{0}-\varepsilon\right), \Omega\right) \neq \nabla_{G}-\operatorname{deg}\left(\nabla_{u} \Phi\left(\cdot, \lambda_{0}+\varepsilon\right), \Omega\right)$, then there is a connected component $\mathcal{C}\left(\lambda_{0}\right)$ of $\operatorname{cl}\left\{(v, \lambda) \in(\mathbb{H} \times \mathbb{R}) \backslash\left(G\left(u_{0}\right) \times \mathbb{R}\right): \nabla_{u} \Phi(u, \lambda)=0\right\}$, containing $G\left(u_{0}\right) \times\left\{\lambda_{0}\right\}$, such that either $\mathcal{C}\left(\lambda_{0}\right) \cap\left(G\left(u_{0}\right) \times\left(\mathbb{R} \backslash\left\{\lambda_{0}\right\}\right)\right) \neq \varnothing$ or $\mathcal{C}\left(\lambda_{0}\right)$ is unbounded. This means that a global bifurcation of solutions of $\nabla_{u} \Phi(u, \lambda)=0$ occurs from the orbit $G\left(u_{0}\right) \times\left\{\lambda_{0}\right\}$.

The proof of this theorem is standard. It is enough to replace in the classical proof (see $[3,19$, 20]) the Leray-Schauder degree by the degree for equivariant gradient operators. Equivariant versions have been formulated in [22] (Theorem 4.9) and [10] (Theorem 3.3). A version for bifurcations from an orbit in finite dimensional representations has been proved in [16] (Theorem 3.4).

Now we turn our attention to relations between the degree theory and the theory of the Conley index. For the Gęba's degree and the Conley index for equivariant maps defined on finite dimensional representations such a relation has been obtained by Gęba [8] (see also Corollary 1 in [11]). We recall it in the following theorem:

Theorem 5.8 Let $\mathbb{V}$ be a finite dimensional $G$-representation and $\varphi \in C^{2}(\mathbb{V}, \mathbb{R})$ a $G$ invariant map such that $G\left(u_{0}\right) \subset(\nabla \varphi)^{-1}(0)$ for some $u_{0} \in \mathbb{V}$. Assume that the orbit $G\left(u_{0}\right)$ is non-degenerate and fix $\Omega \subset \mathbb{V}$ such that $(\nabla \varphi)^{-1}(0) \cap \Omega=G\left(u_{0}\right)$. Then

$$
\nabla_{G}-\operatorname{deg}(\nabla \varphi, \Omega)=\chi_{G}\left(C I_{G}\left(G\left(u_{0}\right),-\nabla \varphi\right)\right) .
$$

\subsection{Eigenspaces of the Laplace operator}

In this subsection we introduce basic properties of the eigenspaces of the Laplace operator (with Neumann boundary conditions) on the ball. More precisely, we study the problem:

$$
\begin{cases}-\Delta u=\beta u & \text { in } B^{N} \\ \frac{\partial u}{\partial v}=0 & \text { on } S^{N-1}\end{cases}
$$

These properties are known, but it is difficult to find a reference in the literature, except for the case $N=2,3$, see for example [1,17]. To make our article self-contained, we sketch here the general case.

Let $\mathcal{H}_{l}^{N}$ denote the linear space of harmonic, homogeneous polynomials of $N$ independent variables, of degree $l$, restricted to the sphere $S^{N-1}$.

Theorem 5.9 The spaces $\mathcal{H}_{l}^{N}$ are irreducible representations of the group $S O(N)$. Furthermore, if $l \geq 1$ then $\mathcal{H}_{l}^{N}$ is a nontrivial representation of $S O(N)$ and for $l=0$ it is a trivial one. Moreover, 


$$
\operatorname{dim} \mathcal{H}_{l}^{N}= \begin{cases}1 & \text { if } N=2, l=0 \\ 2 & \text { if } N=2, l \geq 1 \\ (2 l+N-2) \frac{(N-3+l) !}{l !(N-2) !} & \text { if } N \geq 3, l \geq 0\end{cases}
$$

For the proof of the irreducibility of the spaces $\mathcal{H}_{l}^{N}$ we refer the reader to [12] (Theorem 5.1). The proof of the latter part of the theorem can be found in [25] (Theorem 4.1).

To find eigenspaces of the Eq. (5.3) we write the Laplacian in polar coordinates $r \geq 0$, $\varphi=\left(\varphi_{1}, \ldots, \varphi_{N}\right), 0 \leq \varphi_{i}<\pi$ for $i=1, \ldots, N-1,0 \leq \varphi_{N}<2 \pi$ :

$$
\Delta u=r^{1-N} \frac{\partial}{\partial r}\left(r^{N-1} \frac{\partial u}{\partial r}\right)+\frac{1}{r^{2}} \Delta_{S^{N-1}} u,
$$

where $\Delta_{S^{N-1}}$ is the Laplace-Beltrami operator on $S^{N-1}$. Applying a standard separation of variables $u(\varphi, r)=v(\varphi) \cdot f(r)$ to (5.3), we obtain the system

$$
\begin{aligned}
& -\Delta_{S^{N-1}} v(\varphi)=\mu v(\varphi) \text { on } S^{N-1}, \\
& r^{2} f^{\prime \prime}(r)+(N-1) r f^{\prime}(r)+\left(\beta r^{2}-\mu\right) f(r)=0 \text { on }(0,1), \\
& |f(0)|<\infty, \\
& f^{\prime}(1)=0 .
\end{aligned}
$$

The Eq. (5.4) has solutions only if $\mu$ is an eigenvalue of $-\Delta_{S^{N-1}}$, i.e. $\mu=\mu_{l}:=l(l+N-2)$, $l=0,1, \ldots$, with associated eigenspaces equal $\mathcal{H}_{l}^{N}$, see [25]. Substituting $\mu=\mu_{l}, \rho=\sqrt{\beta} r$ and $f(r)=g(\rho) / \rho^{\frac{N-2}{2}}$ into (5.5), we get the Bessel equation of order $l+\frac{N-2}{2}$ :

$$
\rho^{2} g^{\prime \prime}(\rho)+\rho g^{\prime}(\rho)+\left(\rho^{2}-\left(l+\frac{N-2}{2}\right)^{2}\right) g(\rho)=0 \text { on }(0, \sqrt{\beta}) .
$$

Using (5.6) we obtain that the solution of this equation is $g(\rho)=C_{l} J_{l+\frac{N-2}{2}}(\rho)$, where $C_{l} \in \mathbb{R}$ and $J_{l+\frac{N-2}{2}}$ is the Bessel function of the first kind of order $l+\frac{N-2}{2}$.

Since we are interested only in solutions satisfying (5.7), taking into consideration that $f^{\prime}(r)=\sqrt{\beta}(\sqrt{\beta} r)^{1-\frac{N}{2}}\left(g^{\prime}(\sqrt{\beta} r)-\frac{N-2}{2 \sqrt{\beta} r} g(\sqrt{\beta} r)\right)$, we obtain that $\sqrt{\beta}$ satisfies the equation:

$$
J_{l+\frac{N-2}{2}}^{\prime}(x)-\frac{N-2}{2 x} J_{l+\frac{N-2}{2}}(x)=0 .
$$

For $m \in \mathbb{N}$ we denote by $x_{l m}$ the $m$-th solution of $(5.8)$ in $(0, \infty)$. Put $x_{00}=0$ and $\mathcal{A}_{l}=$ $\left\{\beta_{l m}=x_{l m}^{2}\right\}_{m=1}^{\infty}$ for $l>0$ and $\mathcal{A}_{0}=\left\{\beta_{0 m}=x_{0 m}^{2}\right\}_{m=0}^{\infty}$.

Fact 5.10 From the above considerations:

(1) $\sigma\left(-\Delta ; B^{N}\right)$ is the union of the sets $\mathcal{A}_{l}$,

(2) if $\beta \in \mathcal{A}_{l}$, then $\mathcal{H}_{l}^{N} \subset \mathbb{V}_{-\Delta}(\beta)$, i.e. $\mathcal{H}_{l}^{N}$ is $S O(N)$-equivalent to a subspace of $\mathbb{V}_{-\Delta}(\beta)$. For $\beta \in \sigma\left(-\Delta ; B^{N}\right)$ we have $\mathbb{V}_{-\Delta}(\beta) \approx_{S O(N)} \bigoplus_{l \in\left\{l \geq 0: \beta \in \mathcal{A}_{l}\right\}} \mathcal{H}_{l}^{N}$ (by $\approx_{S O(N)}$ we denote the equivalence relation of $S O(N)$-representations).

Remark 5.11 Since from Theorem 5.9 we have $\operatorname{dim} \mathcal{H}_{0}^{N}=1$ and $\operatorname{dim} \mathcal{H}_{l}^{N}>1$ for $l \geq 1$, it follows that for $\beta \in \sigma\left(-\Delta ; B^{N}\right)$ :

(1) if $\operatorname{dim} \mathbb{V}_{-\Delta}(\beta)>1$, then there exists $l>0$ such that $\mathcal{H}_{l}^{N} \subset \mathbb{V}_{-\Delta}(\beta)$ and thus $\mathbb{V}_{-\Delta}(\beta)$ is a nontrivial $S O(N)$-representation, 
(2) if $\operatorname{dim} \mathbb{V}_{-\Delta}(\beta)=1$, then $\mathbb{V}_{-\Delta}(\beta) \approx_{S O(N)} \mathcal{H}_{0}^{N}$ and therefore it is a trivial representation of $S O(N)$.

Remark 5.12 From Theorem 5.9 we obtain that if $\beta \in \sigma\left(-\Delta ; B^{N}\right)$ and $\beta \notin \mathcal{A}_{0}$, then $\mathbb{V}_{-\Delta}(\beta)^{S O(N)}=\{0\}$.

To illustrate the above description of the eigenspaces, we will look more closely at the cases $N=2,3$.

Suppose that $N=2$. Then, for $l \in \mathbb{N} \cup\{0\}$, the Eq. (5.8) is of the form $J_{l}^{\prime}(x)=0$ and therefore $x_{l m}$ is the $m$-th solution of $J_{l}^{\prime}(x)=0$ in $(0, \infty)$ and $x_{00}=0$.

Fact 5.13 Under the above notation, $\sigma\left(-\Delta ; B^{2}\right)=\bigcup_{l=0}^{\infty} \mathcal{A}_{l}=\left\{\beta_{l m}=x_{l m}^{2}\right\}_{l=1, m=1}^{\infty} \cup$ $\left\{\beta_{0 m}=x_{0 m}^{2}\right\}_{m=0}^{\infty}$ with corresponding eigenvectors given by

(1) $v_{l m}^{1}(r, \phi)=J_{l}\left(x_{l m} r\right) \cos l \varphi$ and $v_{l m}^{2}(r, \phi)=J_{l}\left(x_{l m} r\right) \sin l \varphi$ for $\beta_{l m}$ in the case $l>0$,

(2) $v_{0 m}(r, \phi)=J_{0}\left(x_{0 m} r\right)$ for $\beta_{0 m}$ in the case $l=0$.

Note that from the above fact it follows that $\mathcal{H}_{l}^{2} \approx_{S O(2)} \operatorname{span}\left\{v_{l m}^{1}, v_{l m}^{2}\right\}$ for $l>0$ and $\mathcal{H}_{0}^{2} \approx_{S O(2)} \operatorname{span}\left\{v_{0 m}\right\}$.

Corollary 5.14 Let $\beta \in \sigma\left(-\Delta ; B^{2}\right)$, then

(1) If $\beta \in \mathcal{A}_{l}$ for $l>0$, i.e. $\beta=\beta_{\text {lm }}$ for given $l, m>0$, then $\mathbb{V}_{-\Delta}(\beta)$ is a nontrivial $S O(2)$-representation. Moreover, if $\operatorname{dim} \mathbb{V}_{-\Delta}(\beta)$ is even, then $\mathbb{V}_{-\Delta}(\beta)^{S O(2)}=\{0\}$ and if $\operatorname{dim} \mathbb{V}_{-\Delta}(\beta)$ is odd, then $\mathbb{V}_{-\Delta}(\beta)^{S O(2)} \approx_{S O(2)} \mathcal{H}_{0}^{2}$.

(2) If $\beta \in \mathcal{A}_{0}$, i.e. $\beta=\beta_{0 m}$ for a given $m \in \mathbb{N}$, then $\operatorname{dim} \mathbb{V}_{-\Delta}(\beta)$ is an odd number. Moreover, if $\operatorname{dim} \mathbb{V}_{-\Delta}(\beta)=1$, then $\mathbb{V}_{-\Delta}(\beta) \approx_{S O(2)} \mathcal{H}_{0}^{2}$ is a trivial $S O(2)$-representation.

Suppose now that $N=3$. Then, for $l \in \mathbb{N} \cup\{0\}$, the equation (5.8) is of the form $J_{l+\frac{1}{2}}^{\prime}(x)-\frac{1}{2 x} J_{l+\frac{1}{2}}(x)=0$ and therefore $x_{l m}$ is the $m$-th solution of this equation in $(0, \infty)$ and $x_{00}=0$.

Fact 5.15 Under the above notation, $\sigma\left(-\Delta ; B^{3}\right)=\bigcup_{l=0}^{\infty} \mathcal{A}_{l}=\left\{\beta_{l m}=x_{l m}^{2}\right\}_{l=1, m=1}^{\infty} \cup$ $\left\{\beta_{0 m}=x_{0 m}^{2}\right\}_{m=0}^{\infty}$ with corresponding eigenvectors:

(1) For $\beta_{\text {lm }}$ in the case $l>0$ :

$$
\begin{aligned}
& v_{k m l}^{1}\left(r, \varphi_{1}, \varphi_{2}\right)=\frac{1}{\sqrt{r}} J_{l+\frac{1}{2}}\left(x_{l m} r\right) P_{l k}\left(\cos \varphi_{1}\right) \sin k \varphi_{2}, \\
& v_{k m l}^{2}\left(r, \varphi_{1}, \varphi_{2}\right)=\frac{1}{\sqrt{r}} J_{l+\frac{1}{2}}\left(x_{l m} r\right) P_{l k}\left(\cos \varphi_{1}\right) \cos k \varphi_{2}, \\
& v_{0 m l}\left(r, \varphi_{1}, \varphi_{2}\right)=J_{l+\frac{1}{2}}\left(x_{l m} r\right) P_{l}\left(\cos \varphi_{1}\right),
\end{aligned}
$$

where $k=1, \ldots, l$ and $P_{l k}, P_{l}$ are Legendre functions,

(2) For $\beta_{0 m}: v_{0 m 0}\left(r, \varphi_{1}, \varphi_{2}\right)=J_{\frac{1}{2}}\left(x_{0 m} r\right)$.

From the above fact it follows that $\mathcal{H}_{l}^{3} \approx_{S O(3)} \operatorname{span}\left\{v_{0 m l}, v_{1 m l}^{1}, v_{1 m l}^{2}, \ldots v_{l m l}^{1}, v_{l m l}^{2}\right\}$ for $l>0$ and $\mathcal{H}_{0}^{3} \approx_{S O(3)} \operatorname{span}\left\{v_{0 m 0}\right\}$.

The description of $\mathcal{H}_{l}^{N}$ in the general case can be found in [30] (Chapter IX). 


\section{References}

1. Babič, V.M., Kapilevič, M.B., Mihlin, S.G., Natanson, G.I., Riz, P.M., Slobodeckii, L.N., Smirnov, M.M.: The Linear Equations of Mathematical Physics. Nauka, Moscow (1964). (in Russian)

2. Bartsch, T.: Topological Methods for Variational Problems with Symmetries. Lecture Notes in Mathematics, vol. 1560. Springer, Berlin (1993)

3. Brown, R.F.: A Topological Introduction to Nonlinear Analysis. Birkhäuser, Boston (1993)

4. Dancer, E.N.: On nonradially symmetric bifurcation. J. Lond. Math. Soc. 20(2), 287-292 (1979)

5. Dancer, E.N.: The $G$-invariant implicit function theorem in infinite dimensions II. Proc. R. Soc. Edinb. 102A(3-4), 211-220 (1986)

6. Garza, G.L., Rybicki, S.: Equivariant bifurcation index. Nonlinear Anal. 73(9), 2779-2791 (2010)

7. Gawrycka, J., Rybicki, S.: Solutions of systems of elliptic differential equations on circular domains. Nonlinear Anal. 59(8), 1347-1367 (2004)

8. Gȩba, K.: Degree for Gradient Equivariant Maps and Equivariant Conley Index. Topological Nonlinear Analysis II, pp. 247-272. Birkhäuser, Boston (1997)

9. Gołębiewska, A., Kluczenko, J.: Connected sets of solutions for a nonlinear Neumann problem. Differ. Integral Equ. 30, 838-852 (2016)

10. Gołębiewska, A., Rybicki, S.: Global bifurcations of critical orbits of $G$-invariant strongly indefinite functionals. Nonlinear Anal. 74(5), 1823-1834 (2011)

11. Gołębiewska, A., Rybicki, S.: Equivariant Conley index versus the degree for equivariant gradient maps. Discrete Contin. Dyn. Syst. Ser. S 6(4), 985-997 (2013)

12. Gurarie, D.: Symmetries and Laplacians. Introduction to Harmonic Analysis, Group Representations and Applications North-Holland Mathematics Studies, vol. 174. North-Holland, Amsterdam (1992)

13. Izydorek, M.: Equivariant Conley index in Hilbert spaces and applications to strongly indefinite problems. Nonlinear Anal. TMA 51(1), 33-66 (2002)

14. Kawakubo, K.: The Theory of Transformation Groups. Oxford University Press, Oxford (1991)

15. Kluczenko, J.: Bifurcation and symmetry breaking of solutions of systems of elliptic differential equations. Nonlinear Anal. 75(11), 4278-4295 (2012)

16. Kowalczyk, M.: Bifurcations of critical orbits of invariant potentials with applications to bifurcations of central configurations of the $N$-body problem. Nonlinear Anal. RWA 24, 108-125 (2015)

17. Muchewicz, K., Rybicki, S.: Existence and continuation of solutions for a nonlinear Neumann problem. Nonlinear Anal. 69(10), 3423-3449 (2008)

18. Pérez-Chavela, E., Rybicki, S., Strzelecki, D.: Symmetric Liapunov center theorem. Calc. Var. 56(2), 26 (2017). https://doi.org/10.1007/s00526-017-1120-1

19. Rabinowitz, P.H.: Nonlinear Sturm-Liouville problems for second order ordinary differential equations. Commun. Pure Appl. Math. 23, 939-961 (1970)

20. Rabinowitz, P.H.: Some global results for nonlinear eigenvalue problems. J. Funct. Anal. 7, 487-513 (1971)

21. Rabinowitz, P.H.: Minimax Methods in Critical Point Theory with Applications to Differential Equations. CBMS Regional Conference Series in Mathematics, vol. 65. AMS, Providence (1986)

22. Rybicki, S.: Degree for equivariant gradient maps. Milan J. Math. 73, 103-144 (2005)

23. Rybicki, S., Shioji, N., Stefaniak, P.: Rabinowitz alternative for non-cooperative elliptic systems on geodesic balls. arXiv:1703.08417

24. Rybicki, S., Stefaniak, P.: Unbounded sets of solutions of non-cooperative elliptic systems on spheres. J. Differ. Equ. 259(7), 2833-2849 (2015)

25. Shimakura, N.: Partial Differential Operators of Elliptic Type. Translations of Mathematical Monographs, vol. 99. American Mathematical Society, Providence (1992)

26. Smoller, J., Wasserman, A.: Bifurcation and symmetry-breaking. Invent. Math. 100(1), 63-95 (1990)

27. Stefaniak, P.: Symmetry breaking of solutions of non-cooperative elliptic systems. J. Math. Anal. Appl. 408(2), 681-693 (2013)

28. tom Dieck, T.: Transformation Groups and Representation Theory. Lecture Notes in Mathematics, vol. 766. Springer, Berlin (1979)

29. tom Dieck, T.: Transformation Groups. Walter de Gruyter \& Co, Berlin (1987)

30. Vilenkin, N.J.: Special Functions and the Theory of Group Representation. Translations of Mathematical Monographs, vol. 22. American Mathematical Society, Providence (1988)

31. Wasserman, A.G.: Equivariant differential topology. Topology 8, 127-150 (1969) 